\title{
Existence Theorem for Solitary Waves on Lattices
}

\author{
Gero Friesecke ${ }^{\star}$, Jonathan A. D. Wattis \\ Department of Mathematics, Heriot-Watt University, Edinburgh, EH14 4AS, UK
}

Received: 26 April 1993/in revised form: 12 July 1993

\begin{abstract}
In this article we give an existence theorem for localized travelling wave solutions on one-dimensional lattices with Hamiltonian

$$
H=\sum_{n \in \mathbb{Z}}\left(\frac{1}{2} p_{n}^{2}+V\left(q_{n+1}-q_{n}\right)\right)
$$

where $V(\cdot)$ is the potential energy due to nearest-neighbour interactions. Until now, apart from rare integrable lattices like the Toda lattice $V(\phi)=a b^{-1}\left(e^{-b \phi}+b \phi-1\right)$, the only evidence for existence of such solutions has been numerical. Our result in particular recovers existence of solitary waves in the Toda lattice, establishes for the first time existence of solitary waves in the (nonintegrable) cubic and quartic lattices $V(\phi)=\frac{1}{2} \phi^{2}+\frac{1}{3} a \phi^{3}, V(\phi)=\frac{1}{2} \phi^{2}+\frac{1}{4} b \phi^{4}$, thereby confirming the numerical findings in [1] and shedding new light on the recurrence phenomena in these systems observed first by Fermi, Pasta and Ulam [2], and shows that contrary to widespread belief, the presence of exact solitary waves is not a peculiarity of integrable systems, but "generic" in this class of nonlinear lattices. The approach presented here is new and quite general, and should also be applicable to other forms of lattice equations: the travelling waves are sought as minimisers of a naturally associated variational problem (obtained via Hamilton's principle), and existence of minimisers is then established using modern methods in the calculus of variations (the concentration-compactness principle of P.-L. Lions [3]).
\end{abstract}

\section{Introduction}

Discrete Hamiltonian systems arising in mechanics and physics are notoriously less amenable to analytic techniques than their continuous counterparts. Thus the majority of work on solitary waves on lattices has centred on two areas. Firstly continuum approximations; here the equation of motion reduces to a PDE [4-7] (and hence the

^ Current address: Dept. Mathematics, Carnegie Mellon University, Pittsburgh, PA 15213, USA 
equation govering a travelling wave simply becomes an ODE). And secondly, there are numerical studies $[1,4,5,8]$.

The present paper addresses the issue of existence of such solitary waves on lattices. The Hamiltonian we consider is

$$
H=\sum_{n \in \mathbb{Z}}\left(\frac{1}{2} p_{n}^{2}+V\left(q_{n+1}-q_{n}\right)\right)
$$

where $q_{n}$ denotes the displacement of the $n^{\text {th }}$ lattice site, $p_{n}=\dot{q}_{n}$, and $V(\cdot)$ is the potential energy due to nearest-neighbour interactions. Prototypical are the cubic and quartic systems $V(\phi)=\frac{1}{2} \phi^{2}+\frac{1}{3} a \phi^{3}(a>0)$ and $V(\phi)=\frac{1}{2} \phi^{2}+\frac{1}{4} b \phi^{4}(b>0)$ studied first by Fermi, Pasta and Ulam in their now famous 1955 Los Alamos report [2], see also $[4,1]$. We show that for these model potentials, as well as for a much larger class of interaction potentials, the Hamiltonian system (1) admits an infinite family of solitary waves, parametrised by the total amount of potential energy in the lattice.

Up until now, apart from numerical evidence [1], the only examples of discrete systems known to posses solitary waves have been carefully constructed integrable systems. Of these, only the Toda lattice $V(\phi)=a b^{-1}\left(e^{-b \phi}+b \phi-1\right)$ [9] falls into the class of lattices we consider. While our existence theory in particular recovers existence of solitary waves for the Toda lattice, most interaction potentials will of course not give rise to integrable systems; for example, see [10] for interesting simulations of collisions of highly accurate numerically-determined solitary waves indicating nonintegrability of the cubic and quartic lattice. Our results thus imply that contrary to common belief, there are non-integrable discrete systems which possess exact solitary waves, and show that (unlike integrability) the existence of these waves is in fact "generic" in this class of nonlinear lattices.

Much of the interest in these lattice systems, and especially in solitary waves, has been motivated by the remarkable observations of recurrence made by Fermi-PastaUlam (FPU) [2]. They performed numerical simulations of the cubic and quartic lattice using a sinusoidal initial condition expecting the nonlinearity to promote mixing of energy between the Fourier modes. Over a long period of time mixing did occur, but the energy separated out again, and they saw the initial conditions almost recur. This behaviour was thought to be very surprising at the time and no explanation was known.

In 1965, a similar behaviour was noted by Zabusky and Kruskal [11] in numerical solutions of the $\mathrm{KdV}$ equation. And here, for the first time an explanation was given, namely in terms of the decomposition of initial conditions into the newly discovered soliton waves. These solitons move around the system at different speeds and due to special collision properties regain their shape and speed after interactions. After a certain time the solitons reach their initial placements again, and the initial conditions recur. Following the discovery of the inverse scattering method [12], all these phenomena could later be understood analytically.

However, the $\mathrm{KdV}$ equation is quite different from the (discrete, nonintegrable) systems studied originally by FPU, and despite much interest, the question as to whether such systems also possess solitary waves has been left open until now. Here we show that these lattices do possess exact solitary waves. If the explanation of recurrence is indeed related to solitary waves (as in $\mathrm{KdV}$ and as suggested e.g. in [13]), the next step would be to analyse their stability and interaction properties; however this lies beyond the scope of this article.

Mathematically, the equation governing travelling waves of the discrete system (1) is much more complicated than the ODE obtained for corresponding continuous 
systems: Hamilton's equations give the equation of motion as

$$
\ddot{q}_{n}=V^{\prime}\left(q_{n+1}-q_{n}\right)-V^{\prime}\left(q_{n}-q_{n-1}\right),
$$

and the equation for a travelling wave is obtained by looking for solutions of the form $q_{n}(t)=q(n-c t)$ :

$$
c^{2} q^{\prime \prime}(z)=V^{\prime}(q(z+1)-q(z))-V^{\prime}(q(z)-q(z-1)) .
$$

That is to say, we are dealing with a second order forward-backward differentialdifference equation. Our approach relies on avoiding a direct study of this equation, but instead recasts the problem of finding solutions to (3) as a variational problem. This is performed by exploiting the underlying variational structure of the equations of motion which comes from Hamilton's principle. This idea was also used in [14]. Existence of a minimiser is then established using modern methods in the calculus of variations, namely the concentration-compactness principle of P.-L. Lions [3], together with appropriate a priori estimates on minimising sequences.

The key hypothesis on the interaction potential $V$ needed in the proof is superquadratic growth; conversely, it turns out that for quadratic $V$ the minimum of the variational problem is not attained (and indeed the Hamiltonian (1) does not admit any solitary waves). Here a beautiful, clear and quite nontechnical explanation of why this transition from existence to nonexistence occurs, and of why the "threshold" is given by the exactly quadratic potential, will be seen to be proved by the subadditivity inequalities of P.-L. Lions (inequalities (S) in Sect. 2 below), introduced in a much more general context in [3]. However, before the problem is reduced to inequalities (S), some technical work is needed: in the low energy range (where superquadratic potentials become close to their quadratic approximation and hence close to a potential which does not admit solitary waves), the allowable error in the a priori estimates becomes arbitrarily small, and so we need to "guess" the exact waveform extremely accurately, which we do using the continuum approximation of the lattice. For high energy waves the allowable error is large, making the proof easier. This is worth noting, since up until now the main method of achieving theoretical insight has been the continuum approximation which only works in the low energy limit.

The plan of this paper is as follows. The next section contains a precise statement of our existence result (Theorem 1), a discussion of examples of specific interest (the two FPU lattices, the Toda lattice, and the Lennard-Jones $(6,12)$ potential relevant for the microscopic theory of classical fluids), and an outline of the proof, which does not assume any a priori knowledge about "concentration-compactness" and is written for those interested in the main ideas rather than technical analysis. Section 3, which deals with the existence of a minimiser of the associated variational problem, contains the main technical work and forms the core of this paper. Section 4 is a brief derivation of the travelling wave equation as the Euler-Lagrange equation of the variational problem. We prove monotonicity of the minimising waves in Sect. 5. This fact is not only interesting in its own right, but also needed to complete the proof of our existence theorem. In Sect. 6 we extend our findings to singular interaction potentials such as the Lennard-Jones potential. Nonexistence of solitary waves in lattices with quadratic interaction is established in Sect. 7, complementing our existence theory and justifying the superquadraticity hypothesis required by our variational approach. For the pure quadratic potential the result is simple, since the travelling wave equation reduces to a linear functional differential equation. Finally, Sect. 8 is a note on periodic travelling waves which are also present in these systems and can be found by much simpler methods, using a finite lattice. 


\section{The Existence Theorem}

We pose another problem, that of minimising a functional with a constraint, and show that the solutions of this problem are also solutions of our travelling wave equation (3). This reformulation is obtained by making the travelling wave ansatz in Hamilton's principle, and imposing a constraint on the system, to parametrise the solution family.

To derive the variational formulation, note that the action of a path of the form $q_{n}(t)=q(n-c t)$ in configuration space is

$$
S=\int_{t_{1}}^{t_{2}} \sum_{n \in \mathbb{Z}}\left(\frac{1}{2} \dot{q}_{n}^{2}(t)-V\left(q_{n+1}(t)-q_{n}(t)\right)\right) d t,
$$

and when taken over a time interval of length $1 / c$, reduces to a single integral over the shape function $q(z)$ :

$$
S=\frac{1}{c} \int_{\mathbb{R}}\left(\frac{c^{2}}{2} q^{\prime}(z)^{2}-V(q(z+1)-q(z))\right) d z .
$$

Hence (noting also that any multiple of the constraint may be subtracted from the functional to be minimised) minimising action among paths of the above form is equivalent to the following problem:

Minimise the average kinetic energy

$$
T(q):=\frac{1}{2} \int_{\mathbb{R}} q^{\prime}(z)^{2} d z
$$

subject to the constraint that the average potential energy is fixed

$$
U(q):=\int_{\mathbb{R}} V(q(z+1)-q(z)) d z=K
$$

To be precise, for every path $q_{n}(t)=q(n-c t), T(q)=c^{-2}\langle$ kinetic energy $\rangle$, $U(q)=\langle$ potential energy $\rangle$, and $c T(q)-c^{-1} U(q)=$ action, where $\langle\cdot\rangle$ denotes the average over a time interval of length $1 / c$. In particular, for every travelling wave on the lattice, $U(q)$ is exactly its average potential energy regardless of the wave speed.

The travelling wave equation (3) is easily recognised as the Euler-Lagrange equation of this problem, with the inverse square of the wave speed $\left(c^{-2}\right)$ corresponding to a Lagrange multiplier $\lambda$.

A careful analysis of the above minimisation problem, carried out in Sect. 3 below, leads to the following existence theorem.

Theorem 1. Let $V \in C^{2}(\mathbb{R}), V \geq 0$ in some neighbourhood $(-\delta, \delta)$ of zero, $V(0)=0$, and $V$ superquadratic on at least one side, that is to say

$$
\frac{V(\phi)}{\phi^{2}} \text { increases strictly with }|\phi| \text { for all } \phi \in \Lambda \text {, }
$$

where either $\Lambda=(-\infty, 0)$ or $\Lambda=(0, \infty)$. Then there exists $K_{0} \geq 0$ such that for every $K>K_{0}$ the Hamiltonian system (1) possesses a nontrivial travelling wave with finite kinetic energy and with average potential energy $K$ (i.e. for every $K>K_{0}$ there exists a function $q_{K} \in C^{2}(\mathbb{R})$ with $T\left(q_{K}\right)<\infty$ and $U\left(q_{K}\right)=K$ which solves Eq. (3) for some $c=c_{K} \neq 0$.) If in addition $V$ satisfies either

$$
V^{\prime \prime}(0)=0
$$


or the following nondegeneracy condition at zero:

$$
V(\phi)=\frac{1}{2} V^{\prime \prime}(0) \phi^{2}+\varepsilon|\phi|^{p}+o\left(\phi^{p}\right) \quad \text { as } \quad \phi \in \Lambda, \phi \rightarrow 0
$$

for some $\varepsilon>0$ and $2<p<6$, then $K_{0}$ can be taken to be zero, that is to say nontrivial travelling waves $q_{K}$ with average potential energy $K$ exist for all $K>0$. Moreover regardless of whether or not $(\mathrm{H} 2 \mathrm{a}),(\mathrm{H} 2 \mathrm{~b})$ hold, these travelling waves $q_{K}$ have the following properties:

(P1) They are monotone functions, increasing (i.e. expansion waves) if $\Lambda=\mathbb{R}^{+}$and decreasing (i.e. compression waves) if $\Lambda=\mathbb{R}^{-}$.

(P2) They are localised, in the sense that $\phi_{K}(z):=q_{K}(z+1)-q_{K}(z) \rightarrow 0$ as $z \rightarrow \pm \infty$.

(P3) They are supersonic; that is to say their wave speeds $c_{K}$ satisfy $c_{K}^{2}>V^{\prime \prime}(0)$.

(P4) They minimise the kinetic energy $T(q)$ among all functions $q$ in the Sobolev space $W_{\text {loc }}^{1,2}(\mathbb{R})$ having the same "symmetrised" potential energy $\tilde{U}(q):=\int_{\mathbb{R}} \tilde{V}(q(z+1)-$
$q(z)) d z$, where

$$
\tilde{V}(\phi):= \begin{cases}V(\phi), & \phi \in \Lambda \\ V(-\phi), & \phi \notin \Lambda .\end{cases}
$$

Discussion. Conversely, we prove that for exactly quadratic potentials, no solitary waves exist (Sect. 6), thereby justifying hypothesis (H1). Hypothesis (H2) (and in particular the bound $p<6$ ) is open to improvement, at the expense of more technical work, see the proof of Proposition 3; however, since $(\mathrm{H} 2)$ is a "generic" consequence of (H1) (it holds for every smooth potential which satisfies (H1) and whose cubic, quartic and quintic Taylor coefficients at zero do not vanish simultaneously), we have not pursued this, to keep the proof as simple as possible.

Applying the theorem to the Tolda Lattice $\left(V(\phi)=a b^{-1}\left(e^{-b \phi}+b \phi-1\right), a b>0\right)$, we find solitary waves on one side of the potential well only (since the Toda potential is superquadratic on one side of the potential well $\phi=0$ and subquadratic (i.e. $\frac{V(\phi)}{\phi^{2}}$ decreasing with $|\phi|)$ on the other; i.e. if $b>0$ the solitary waves have $\phi \leq 0$ and vice versa. This agrees, of course, with the findings of Toda [9], while being derived from a completely different method.

A similar lattice is given by the cubic polynomial interaction, $V(\phi)=\frac{1}{2} \phi^{2}+$ $\frac{1}{3} a \phi^{3}(a \neq 0)$. Here again we have existence of solitary waves, confirming the numerical observations in [1]. The sign of the waves depends on the sign of the parameter $a$, (if $a>0$, then $\phi \geq 0$ ).

If we turn to the quartic lattice, $V(\phi)=\frac{1}{2} \phi^{2}+\frac{1}{4} b \phi^{4}$, we find a different story. If $b>0$ then solitary waves exist either side of $\phi=0$ (again confirming the findings in [1]). However as $b$ is reduced through zero, our existence result no longer applies, and at $b=0$, we have non-existence of solitary waves. Further, we conjecture that for $b<0$ there is no solitary wave. This is supported by the fact that the variational problem no longer attains its infimum (see Proposition 2), and by some formal asymptotics; though a rigorous proof has yet to be found. The vanishing of solitary waves as $b$ passes through zero can be thought of as a phase transition, and is not particular to the quartic lattice. This phenomenon should occur for any lattice where the interaction potential passes from super- to subquadratic (or where the coupling force weakens from super- to sublinear) as a parameter is varied. 
As a final example, consider the paradigm interaction potential of the microscopic theory of classical fluids, the Lennard-Jones $(6,12)$ potential $V_{L J}(r)=a r^{-12}-$ $b r^{-6}(a, b>0)$, where $r$ is the distance between particles. To fit our setting, we let $d=(2 a / b)^{1 / 6}$ - the equilibrium distance, change variables $r=d+\phi$ (so that $\phi$ denotes the deviation of the interparticle distance from the equilibrium distance) and shift the energy scale to make the minimum energy equal to zero, giving $V(\phi)=a\left((d+\phi)^{-6}-d^{-6}\right)^{2}$. Here $\phi$ is of course restricted to values $\phi>-d$. With this choice of $V$, Eq. (2) represents the evolution at the microscopic level of a one-dimensional classical fluid in the nearest neighbour approximation. To check super- or subquadraticity, calculate

$$
\begin{aligned}
\left(\frac{V(\phi)}{\phi^{2}}\right)^{\prime} & =\frac{V^{\prime}(\phi) \phi-2 V(\phi)}{\phi^{3}} \\
& =-\frac{1}{\phi^{3}} \frac{2 a}{d^{12}(d+\phi)^{13}}\left((d+\phi)^{6}-d^{6}\right)\left((d+\phi)^{7}-\left(d^{7}+7 d^{6} \phi\right)\right) .
\end{aligned}
$$

Clearly, for $\phi \neq 0$ the second and fourth factors are always positive, the first and third factors have the same sign as $\phi$, hence $\left(\frac{V(\phi)}{\phi^{2}}\right)^{\prime}<0$ for all $\phi \neq 0$. Thus the Lennard-Jones potential is superquadratic on the left side of the potential well, and subquadratic on the right side. Correspondingly, we obtain existence of compression waves (i.e. $\phi \leq 0$ ), but not of expansion waves. (The precise existence result for the Lennard-Jones potential and the technical details needed to adapt Theorem 1 to deal with the singularity of $V$ can be found in Sect. 6.) These findings correspond nicely to the situation in macroscopic, one-dimensional fluid models such as $\mathrm{KdV}$, where negative solitons exist but positive ones don't, and to the experimental behaviour of water in a narrow channel, where the observed solitary waves are compression waves rather than expansion waves (for the first scientific account of these waves, see Scott Russell's remarkable 1844 paper [15]).

The generality of Theorem 1 shows that solitary waves are generic in lattice systems with a superquadratic potential energy. The existence of solitons (that is, solitary waves which regain their shape after interactions) on the other hand is a very special property of a system. The systems of FPU (i.e., the cubic and the quartic lattice) fall into the former category, with no known special properties. Thus it seems unlikely that the explanation of recurrence in these systems is due to solitary wave interactions alone. However it may play an important part, and has already been postulated [13]. A full explanation of the mechanism would require an analysis of the interaction of solitary waves with each other, and with linear waves. This has yet to be attempted, but some initial results suggesting the linear stability of solitary waves have been found [16].

Outline of Proof. The key step in the existence proof is to solve the variational problem, that is, to show there exist minimisers of $T(q)$ subject to the constraint that the "symmetrised" potential energy is prescribed, $\tilde{U}(q)=K,(K>0$ fixed throughout the proof). Once this is done, the rest of the proof is more less straightforward: we then derive the Euler-Lagrange equation to this problem and demonstrate positivity of the occurring Lagrange multiplier $\lambda$ so as to be able to identify the Euler-Lagrange equation with the travelling wave equation (2) and $\lambda$ with the inverse square of the wave speed $c^{-2}$ (Sect. 4), and finally show monotonicity of minimisers which implies that (after choosing a minimiser of the desired sign) its difference function 
$\phi_{K}(z):=q_{K}(z+1)-q_{K}(z)$ only attains values on the side of the potential well, where $\tilde{V}=V$, so that it is a travelling wave for the original "unsymmetrised" Hamiltonian (Sect. 5).

The core of the proof, the existence of minimisers, is dealt with in Sect. 3. Due to the unboundedness of the domain of integration, this is delicate and cannot be inferred by standard "soft" methods of the calculus of variations (e.g. weak convergence/weak lower semicontinuity arguments); indeed we will see that for quadratic or subquadratic interaction potentials (i.e. weak coupling) the minimum is not attained. At the expense of boring experts in variational methods, let us explain what the main difficulty is (and how to interpret the failure of the "soft" approach physically), and then recall the gist of P.-L. Lions' concentration-compactness method which was devised to attack this difficulty. As already emphasised in the Introduction, this method should not be regarded as just a technical tool, but P.-L. Lions' subadditivity inequalities (S) below are essential for understanding why the behaviour for super- and sub-quadratic potentials is different and why the "threshold" is given by the exactly quadratic potential.

According to the standard approach of the calculus of variations, one would take a minimising sequence $\left\{q_{n}\right\}$ of $T$ subject to the constraint ${ }^{1}$ that $U\left(q_{n}\right) \equiv K$, then (since $T\left(q_{n}\right)$ is bounded) $q_{n}^{\prime}$ is a bounded sequence in $L^{2}(\mathbb{R})$, and we can pass to a weakly convergent subsequence. Since the limit possesses a primitive $q$, we can write $q_{n_{k}}^{\prime} \rightarrow q^{\prime}$ weakly in $L^{2}(\mathbb{R})$. By weak lower semicontinuity of the norm in $L^{2}(\mathbb{R}), T(q) \leq \inf _{U(q)=K} T(q)$, and equality would hold, i.e. $q$ would be a minimiser, provided we could show $U(q)=K$ (survival of the constraint). It is exactly at this last point where the standard approach fails: due to unboundedness of the domain, the constraint is discontinuous with respect to weak convergence of the $q_{n_{k}}^{\prime}$, and it is easy to write down minimising sequences where the constraint is indeed lost in the limit. One class of examples where this happens, even if the minimum is attained, arises from the translation invariance of $T$ and $U$ : simply take $q_{n}$ to be a sequence of translates $q(\cdot+n)$ of a minimiser $q$. Then the $q_{n}^{\prime}$ converge weakly to zero in $L^{2}(\mathbb{R})$, and thus $U(q)=0\left(\neq K=\lim U\left(q_{n}\right)\right)$. Another mechanism of loss of the constraint, typically associated with nonattainment of the minimum and known to occur in various precedents of variational problems similar to the one studied here, would be the flattening and spreading out of a part of, or the whole, potential energy integrand $V\left(q_{n}(\cdot+1)-q_{n}(\cdot)\right)$, this phenomenon corresponding physically to excess energy/mass/charge carried by the sequence $\left\{q_{n}\right\}$ moving off to infinity as $n \rightarrow \infty$. A collection of situations where this happens can be found in [17], interesting examples being the Becker-Döring equations describing the kinetics of cluster growth [18] and the Thomas-Fermi model of atoms and molecules [19].

Against this background, in his fundamental article [3], P.-L. Lions suggested a general framework in which to analyse such problems, based on the following two key heuristic ideas (which he made rigorous for some nontrivial examples, and which - adapted to the present context - take the following form). First, there is a complete list of possible ways in which the constraint can be lost. Namely, every minimising sequence $\left\{q_{n}\right\}$ possesses a subsequence which satisfies one of the following three possibilities:

\footnotetext{
1 For the remainder of this section, we shall drop the "tilde" above $V$ and $U$, and let $V$ be an arbitrary $C^{2}$ potential with $V(0)=0$ and $V>0$ elsewhere, for the moment not required to satisfy $(\mathrm{H} 1)$ or $(\mathrm{H} 2)$
} 
(i) (compactness) After replacing the $q_{n}$ by appropriately chosen translates $q_{n}(\cdot+$ $a_{n}$ ), the constraint $U\left(q_{n}\right)=K$ is preserved when passing to a weak limit (note that by a retranslation one can prevent loss of the constraint by the region of interest being shifted away to infinity as in the first example above),

(ii) (vanishing) the sequence of integrands $V\left(q_{n}(\cdot+1)-q_{n}(\cdot)\right)$ converges to zero by flattening and spreading out, or

(iii) (splitting) the integrands "split" (in a sense, of course, to be made precise) into at least two spatially separate parts, with the distance between them tending to infinity as $n \rightarrow \infty$, and the total amount of potential energy contained in each part converging, respectively to $\alpha \in(0, K)$ and $K-\alpha$.

(This is stated rigorously in Lemma 2, with the notion of "compactness" given above being implied by the rigorous notion via the proof of Proposition 1.) Second, following [3] alternative (iii) can be excluded by considering the minimum energy $T_{K}=\inf \{T(q): U(q)=K\}$ as a function of $K$ and verifying the subadditivity inequalities

$$
T_{K}<T_{\alpha}+T_{K-\alpha} \text { for all } \alpha \in(0, K)
$$

In fact, no minimising sequence can split if and only if the above inequalities hold true. Unlike the list of possible ways of losing the constraint, this second idea is on a heuristic level obvious, and it is also not hard to make it rigorous (see the proof of Lemma 3a). (In our case of solitary waves inequalities (S) - whenever they hold true - suggest that it is unfavourable in the sense of least action for a minimising wave to split into two waves. This could be taken as a type of stability of the wave; numerical simulations suggest that these waves are indeed quite stable. In the related, but analytically much simpler problem of standing waves in the continuous nonlinear Schrödinger equation, inequalities (S) have indeed been made the basis of a rigorous stability result [20].)

Now for our special problem, a similar strategy is possible to exclude "vanishing:" no minimising sequence can vanish if and only if the minimum kinetic energy satisfies the bound

$$
T_{K}<\frac{K}{V^{\prime \prime}(0)}
$$

(see Lemma 3b). Here the expression on the right-hand side turns up as the limit $\varepsilon \rightarrow 0$ of the infima $T_{K, \varepsilon}:=\inf \left\{T(q): U(q)=K, \sup _{z \in \mathbb{R}}|q(z+1)-q(z)| \leq \varepsilon\right\}$, that is to say, the infima of $T$ over functions $q$ whose potential energy density is more and more flat and spread out.

Equipped with these ideas or tools (which reduce our problem to analysing whether or not inequalities (E) and (S) hold true), it is now possible to establish existence of a minimiser for superquadratic interaction potentials and nonexistence for subquadratic potentials. Here the key to understanding why the behaviour of these two cases is different is to look at P.-L. Lions' subadditivity inequalities (S): a relatively simple scaling argument (using the fact that the kinetic energy $T$ scales quadratically) shows that inequalities (S) fail for subquadratic interaction potentials, but hold true in the superquadratic case provided we can exclude vanishing; the reader is invited to have a brief look at the proof (proof of Lemma 4). The main technical work in proving that in the superquadratic case minimisers indeed exist goes into establishing the above trichotomy, and into verifying (E) so as to exclude vanishing.

In order to establish (E), we have to "guess" a sufficiently good aproximate minimiser which has lower energy than the limit $K / V^{\prime \prime}(0)=: T_{K, 0}$ of the $T_{K, \varepsilon}$. 


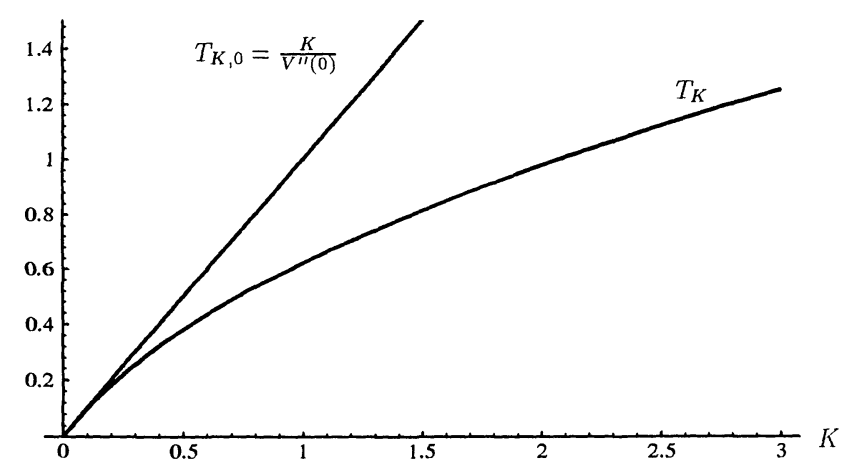

Fig. 1. The minimum kinetic energy $T_{K}$ (note its subadditivity!) and the "vanishing-energy" $T_{K, 0}$, plotted here for the quartic lattice $V(\phi)=\frac{1}{2}\left(\phi^{2}+\phi^{4}\right)$. In Sect. 3 we show that these curves always become tangent at $K=0$, for any potential $V \in C^{2}(\mathbb{R})$ satisfying $V \geq 0, V(0)=0, V^{\prime \prime}(0) \neq 0$

If the prescribed potential energy $K$ is large, then it suffices to choose a simple piecewise linear approximation of the actual travelling wave (see Proposition 2), but in the low-energy range these and similar crude guesses will have higher energy than $T_{K, 0}$. The reason why much finer approximations are needed in the lowenergy range is the following: If $K$ is small, then (by Lemma 1(a) and Lemma 1(d)) so is $\sup |q(z+1)-q(z)|$ whenever $T(q)$ is near its infimum; but for small $\phi$, $V(\phi) \approx \frac{1}{2} V^{\prime \prime}(0) \phi^{2}$, i.e. the potential behaves approximately like a quadratic, and for quadratic $V$ the minimum is not attained. That is to say, the smaller the prescribed potential energy, the "closer" we are to a situation where the minimum is not attained (see Fig. 1).

The fact that comes to our rescue is that in the low-energy range, the continuum approximation of the lattice is valid. For example for our two model potentials $V(\phi)=\frac{1}{2} \phi^{2}+\frac{1}{3} a \phi^{3}(a>0), V(\phi)=\frac{1}{2} \phi^{2}+\frac{1}{4} b \phi^{4}(b>0)$ the standard continuum approximations are, respectively, the Boussinesq and modified Boussinesq equation, for which travelling waves exist and are known explicitly; e.g. for the Boussinesq equation $\phi_{t t}=\phi_{x x}+\frac{1}{12} \phi_{x x x x}+\left(a \phi^{2}\right)_{x x}$, these waves have the form $q=\Lambda \tanh (\beta z)$, where $\phi=q^{\prime}$. Using these waves as "guesses" for the low-energy waves of the corresponding discrete systems, one then indeed finds that their energy is lower than $T_{K, 0}$ (Proposition 3). This excludes vanishing, and completes the existence proof.

The method underlying our proof and explained here is quite general and should also be applicable to other forms of lattice equations.

\section{Analysis of the Variational Problem}

This section is concerned with the existence of minimisers to our variational problem (4), and forms the core of this paper. The natural class of functions in which to seek minimisers is the domain of $T,\left\{q \in W_{\mathrm{loc}}^{1,2}(\mathbb{R}): \int_{\mathbb{R}} q^{\prime}(z)^{2} d z<\infty\right\}$. Adding the normalisation condition that $q(0)=0$ makes domain $T$ into a Hilbert space

$$
X:=\left\{q \in W_{\mathrm{loc}}^{1,2}(\mathbb{R}): \int_{\mathbb{R}} q^{\prime}(z)^{2} d z<\infty ; q(0)=0\right\},
$$


with the obvious norm and inner product $\langle q, p\rangle=\int_{\mathbb{R}} q^{\prime} p^{\prime},\|q\|=\left(\int_{\mathbb{R}} q^{\prime 2}\right)^{1 / 2}$. We then
seek to minimise $T$ on the subsets

$$
\mathscr{A}_{K}:=\left\{q \in X: V(q(\cdot+1)-q(\cdot)) \in L^{1}(\mathbb{R}) ; U(q)=K\right\} \text {. }
$$

The following elementary lemma states that for the physically relevant potentials $V, V(q(\cdot+1)-q(\cdot))$ automatically lies in $L^{1}(\mathbb{R})$ for all $q \in X$, collects together various other basic properties of $X$, the $\mathscr{A}_{K}$, and the minimum values

$$
T_{K}:=\inf _{\mathscr{C}_{K}} T
$$

for later use.

Lemma 1. Assume $V \in C^{2}(\mathbb{R}), V(0)=0, V \geq 0, V>0$ in some neighbourhood $(-\delta, \delta) \backslash\{0\}$ of zero. Then:

(a) For every $q \in X$, the corresponding difference function $\phi(z):=q(z+1)-q(z)$ has the properties: $\phi \in C^{0}(\mathbb{R})$, $\sup _{z \in \mathbb{R}}|\phi(z)| \leq\|q\|, \phi(z) \rightarrow 0$ as $z \rightarrow \pm \infty$.

(b) The map $q \mapsto V(\phi)$ maps $X$ into $L^{1}(\mathbb{R})$, and is continuous between these spaces. In particular $U$ is defined on the whole of $X$ and is continuous, and $\mathscr{A}_{K}=\{q \in$ $X: U(q)=K\}$.

(c) The sets $\mathscr{C}_{K}$ are nonempty for all $K \in[0, \infty)$, hence the minimum values $T_{K}$ are well-defined.

(d) $T_{K}$ is a monotone increasing and continuous function of $K \in[0, \infty)$, and $T_{K}>0$ for all $K>0$.

Proof. (a) follows immediately from the Sobolev embedding $W_{\text {loc }}^{1,2}(\mathbb{R}) \hookrightarrow C^{0}(\mathbb{R})$, together with the elementary estimate

$$
|\phi(z)| \leq \int_{z}^{z+1}\left|q^{\prime}\right| \leq\left(\int_{z}^{z+1}\left(q^{\prime}\right)^{2}\right)^{1 / 2}
$$

which shows $\phi(z) \rightarrow 0$ as $z \rightarrow \pm \infty$ and $|\phi(z)| \leq\|q\|$.

To show (b), fix $C>0$ and consider $q_{1}, q_{2} \in X,\left\|q_{i}\right\|<C$. Let $\phi_{i}:=$ $q_{i}(\cdot+1)-q_{i}(\cdot)$. By (a), $\max \left\{\left|\phi_{1}(z)\right|,\left|\phi_{2}(z)\right|\right\} \leq C$ for all $c$, hence (letting $\sup _{[-C, C]}\left|V^{\prime \prime}\right|=: C_{1}$ and using $\left.V^{\prime}(0)=0\right)$

$$
\begin{aligned}
\int_{\mathbb{R}}\left|V\left(\phi_{2}\right)-V\left(\phi_{1}\right)\right| & \leq C_{1} \int_{\mathbb{R}}\left(\left|\phi_{1}\right|+\left|\phi_{2}\right|\right)\left|\phi_{2}-\phi_{1}\right| \\
& \leq C_{1}\left(\left\|\phi_{1}\right\|_{L^{2}(\mathbb{R})}+\left\|\phi_{2}\right\|_{L^{2}(\mathbb{R})}\right)\left\|\phi_{2}-\phi_{1}\right\|_{L^{2}(\mathbb{R})} .
\end{aligned}
$$

Now the terms on the right-hand side can be estimated using (5) and the elementary integral identity

$$
\int_{\mathbb{R}} \int_{t-\tau}^{t} f(s) d s d t=\tau \int_{\mathbb{R}} f(t) d t,
$$


valid for any $f \in L^{1}(\mathbb{R})$ :

$$
\int_{\mathbb{R}} \phi_{i}^{2} \leq \int_{\mathbb{R}} \int_{z}^{z+1}\left(q_{i}^{\prime}\right)^{2}=\int_{\mathbb{R}}\left(q_{i}^{\prime}\right)^{2}=\left\|q_{i}\right\|^{2}<C^{2},
$$

and analogously $\int_{\mathbb{R}}\left(\phi_{2}-\phi_{1}\right)^{2} \leq\left\|q_{2}-q_{1}\right\|^{2}$. Consequently

$$
\int_{\mathbb{R}}\left|V\left(\phi_{2}\right)-V\left(\phi_{1}\right)\right| \leq 2 C_{1} C\left\|q_{2}-q_{1}\right\|
$$

proving (b).

To show (c), pick $\Lambda_{0}>0$ s.t. $V\left(\Lambda_{0}\right)>0$, and consider the functions

$$
q_{\Lambda, L}(z):= \begin{cases}0, & z \leq 0 \\ \Lambda z, & z \in[0, L] \\ \Lambda L, & z>L\end{cases}
$$

$(\Lambda \geq 0, L \geq 1)$. Clearly $q_{\Lambda, L} \in X$, and $U\left(q_{\Lambda, L}\right)=(L-1) V(\Lambda)+2 \int_{0}^{1} V(\Lambda z) d z$, in particular $U\left(q_{\Lambda_{0}, L}\right) \rightarrow \infty$ as $L \rightarrow \infty$. Now by the continuity of $U, U\left(q_{\Lambda, 1}\right)(\Lambda \in$ $\left.\left[0, \Lambda_{0}\right]\right)$ attains each intermediate value $\in\left[0, U\left(q_{\Lambda_{0}, 1}\right)\right]$, and $U\left(q_{\Lambda_{0}, L}\right)(L \in[1, \infty))$ attains each intermediate value $\in\left[U\left(q_{\Lambda_{0}, 1}\right), \infty\right)$, proving (c).

Next, we show that $T_{K}$ is nondecreasing with $K$. Let $\alpha \leq K$, and take $q \in \mathscr{A}_{K}$. Since $U(\Lambda q)=0$ at $\Lambda=0$ and $=K$ at $\Lambda=1$, there exists $\Lambda_{0} \in[0,1]$ such that $U\left(\Lambda_{0} q\right)=\alpha$. Hence

$$
T_{\alpha} \leq T\left(\Lambda_{0} q\right)=\Lambda_{0}^{2} T(q) \leq T(q) .
$$

Since $q \in \mathscr{A}_{K}$ was arbitrary, $T_{\alpha} \leq T_{K}$, establishing monotonicity. We now show $T_{K}$ is continuous. In view of the monotonicity of $T_{K}$, it suffices to show that there exists $\eta(\delta) \rightarrow 0(\delta \rightarrow 0)$ such that $T_{K+\delta}-T_{K} \leq \eta(\delta)$ for all $K \geq 0, \delta>0$. Fix $K$ and $\delta$. Given $\varepsilon>0$, pick $q_{K} \in \mathscr{A}_{K}$ such that $T\left(q_{K}\right) \leq T_{K}+\varepsilon$, and consider the function

$$
q(z):= \begin{cases}q_{K}(z), & z \leq a \\ q_{K}(a)+q_{\Lambda, 1}(z-a-1), & z \geq a\end{cases}
$$

(with $q_{\Lambda, 1}$ as defined in (8) and $a$ to be specified shortly). Then $q \in X$, and

$$
U(q)=\int_{-\infty}^{a-1} V\left(\phi_{K}\right)+\int_{a-1}^{a} V(\phi)+U\left(q_{\Lambda, 1}\right)
$$

where $\phi_{K}:=q_{K}(\cdot+1)-q_{K}(\cdot), \phi:=q(\cdot+1)-q(\cdot)$. Clearly the first term tends to $K$ and the second term to zero as $a \rightarrow \infty$, thus $U(q) \rightarrow K+U\left(q_{\Lambda, 1}\right)=K+2 \int_{0}^{1} V(\Lambda z) d z$ as $a \rightarrow \infty$. Assume without loss $\delta \in\left(0, \frac{1}{2} U\left(q_{\Lambda_{0}, 1}\right)\right)$, and define $\Lambda_{1}(\delta):=\inf \{\Lambda>$ $\left.0: U\left(q_{\Lambda, 1}\right)=2 \delta\right\}$. Now by continuity of $U$, there exist $a \in \mathbb{R}$ and $\Lambda \leq \Lambda_{1}(\delta)$ such that $U(q)=K+\delta$, and hence

$$
T_{K+\delta}-T_{K} \leq T(q)-T_{K} \leq T(q)-T\left(q_{K}\right)+\varepsilon \leq T\left(q_{\Lambda, 1}\right)+\varepsilon \leq \frac{1}{2} \Lambda_{1}(\delta)^{2}+\varepsilon .
$$


Now $\Lambda_{1}(\delta)$ is independent of $K$ and $\varepsilon$ and tends to zero as $\delta \rightarrow 0$ (because $V>0$ near zero). Since $\varepsilon$ was arbitrary, this establishes continuity. Finally, the fact that $T_{K}>0$ for $K>0$ follows immediately from setting $q_{2}=0$ in (7). The lemma is proved.

Now the starting point for addressing the question of existence or nonexistence of minimisers is the concentration-compactness principle of P.-L. Lions [3]. As pointed out in that article, this principle is only formal and an appropriate variant of it has to be rigorously derived for each problem. Here the principle takes the following form:

Lemma 2 (concentration-compactness; compare [3, Lemma III.1]). Assume that the potential $V$ satisfies $V \in C^{2}(\mathbb{R}), V(0)=0, V \geq 0$. Let $\left\{q^{k}\right\}$ be a sequence in $W_{\text {loc }}^{1,2}(\mathbb{R})$ such that

$$
\sup _{k}\left\|\left(q^{k}\right)^{\prime}\right\|_{L^{2}(\mathbb{R})} \leq C, \quad \int_{\mathbb{R}} V^{k} \equiv K,
$$

where $K>0$ is fixed and $V^{k}(z)=V\left(q^{k}(z+1)-q^{k}(z)\right)$. Then there exists a subsequence, again denoted $q^{k}$, satisfying one of the following three possibilities:

(i) (compactness) $\exists y_{k} \in \mathbb{R}$ s.t. $V^{k}\left(\cdot+y_{k}\right)$ is "tight," i.e. $\forall \varepsilon>0 \exists R<\infty$ s.t. $\int_{\mathbb{R} \backslash B_{R}\left(y_{k}\right)} V^{k} \leq \varepsilon \forall k$.

(ii) (vanishing) $\lim _{k \rightarrow \infty} \sup _{y \in \mathbb{R}} \int_{B_{R}(y)} V^{k}=0 \forall R<\infty$.

(iii) (splitting) $\exists \alpha \in(0, K)$ s.t $\forall \varepsilon>0, \exists k_{0} \geq 1$ and $q_{1}^{k}, q_{2}^{k} \in W_{\text {loc }}^{1,2}(\mathbb{R}),\left\|q_{j}^{k}\right\|_{L^{2}(\mathbb{R})}<\infty$, s.t for $k \geq k_{0}$,

$$
\begin{gathered}
\left\|V^{k}-\left(V_{1}^{k}+V_{2}^{k}\right)\right\|_{L^{1}(\mathbb{R})} \leq \varepsilon, \quad\left|\int_{\mathbb{R}} V_{1}^{k} d x-\alpha\right| \leq \varepsilon, \\
\left|\int_{\mathbb{R}} V_{2}^{k} d x-(K-\alpha)\right| \leq \varepsilon, \\
\frac{1}{2} \int_{\mathbb{R}}\left(\left(q^{k}\right)^{\prime}\right)^{2}-\frac{1}{2} \int_{\mathbb{R}}\left(\left(q_{1}^{k}\right)^{\prime}\right)^{2}-\frac{1}{2} \int_{\mathbb{R}}\left(\left(q_{2}^{k}\right)^{\prime}\right)^{2} \geq 0, \\
\operatorname{dist}\left(\operatorname{supp}\left(q_{1}^{k}\right)^{\prime}, \operatorname{supp}\left(q_{2}^{k}\right)^{\prime}\right) \rightarrow \infty,
\end{gathered}
$$

where $V_{j}^{k}(z)=V\left(q_{\jmath}^{k}(z+1)-q_{j}^{k}(z)\right)$.

Technical Remark. Under the stronger assumption that $\left\{q^{k}\right\}$ be bounded in $W^{1,2}(\mathbb{R})$ (and for simple local constraints such as $\int_{\mathbb{R}}\left(q^{k}\right)^{2} \equiv K$ ) this is proved in [3, Lemma III.1]. The construction in [3] of $q_{1}^{k}, q_{2}^{k}$ via cutoff-functions does not work here, due to the lack of an a-priori bound on $\left\|q^{k}\right\|_{L^{2}(\mathbb{R})}$.

Proof. The first part of the proof goes as in [3]. We use the concentration functions of the measures $V^{k} d x, Q_{k}(R):=\sup _{y \in \mathbb{R}} \int_{B_{R}(y)} V^{k}$. By passing to a subsequence we may assume $Q_{k}(R) \rightarrow Q(R)$ pointswise $\forall R \geq 0$ for some nonnegative nondecreasing funtion $Q$, we let $\alpha:=\lim _{R \rightarrow \infty} Q(R) \in[0, K]$, and we note $\alpha=0 \Rightarrow$ (ii), $\alpha=K \Rightarrow$ (i). So all we have to show is " $\alpha \in(0, K) \Rightarrow$ (iii)." 
Fix $\varepsilon>0$, and choose $R$ such that $Q(R-1)>\alpha-\varepsilon$. Then for $k$ large enough, $Q_{k}(R-1)>\alpha-\varepsilon$, hence $\exists y_{k} \in \mathbb{R}$ such that

$$
\int_{B_{R-1}\left(y_{k}\right)} V^{k}>\alpha-\varepsilon
$$

Furthermore since $\lim _{R \rightarrow \infty} Q(R)=\alpha$, we can find $R^{k} \rightarrow \infty$ such that

$$
Q_{k}\left(R^{k}+1\right)<\alpha+\varepsilon
$$

Now for $R_{1}^{k} \in\left[R, R+\frac{1}{3}\left(R^{k}-R\right)\right], R_{2}^{k} \in\left[R+\frac{2}{3}\left(R^{k}-R\right), R^{k}\right],\left(R_{1}^{k}, R_{2}^{k}\right.$ to be specified later), define

$$
\begin{aligned}
& q_{1}^{k}(z):= \begin{cases}q^{k}\left(y_{k}-R_{1}^{k}\right), & z \leq y_{k}-R_{1}^{k} \\
q^{k}(z), & z \in B_{R_{1}^{k}}\left(y_{k}\right) \\
q^{k}\left(y_{k}+R_{1}^{k}\right), & z \geq y_{k}+R_{1}^{k}\end{cases} \\
& q_{2}^{k}(z):= \begin{cases}q^{k}(z)-q^{k}\left(y_{k}-R_{2}^{k}\right), & z \leq y_{k}-R_{2}^{k} \\
0, & z \in B_{R_{2}^{k}}\left(y_{k}\right) \\
q^{k}(z)-q^{k}\left(y_{k}+R_{2}^{k}\right), & z \geq y_{k}+R_{2}^{k} .\end{cases}
\end{aligned}
$$

Then

$$
\left.\frac{1}{2} \int_{\mathbb{R}}\left(\left(q^{k}\right)^{\prime}\right)^{2}-\frac{1}{2} \int_{\mathbb{R}}\left(\left(q_{1}^{k}\right)^{\prime}\right)^{2}-\frac{1}{2} \int_{\mathbb{R}}\left(\left(q_{2}^{k}\right)^{\prime}\right)^{2}=\frac{1}{2} \int_{B_{R_{2}}\left(y_{k}\right) \backslash B_{R_{1}}\left(y_{k}\right)}\left(q^{k}\right)^{\prime}\right)^{2} \geq 0,
$$

and $\operatorname{dist}\left(\operatorname{supp}\left(q_{1}^{k}\right)^{\prime}, \operatorname{supp}\left(q_{2}^{k}\right)^{\prime}\right) \rightarrow \infty$. It remains to verify the other statements of (iii). These can be achieved by an appropriate choice of $R_{1}^{k}$ and $R_{2}^{k}$, as follows. First of all, for every $q \in W_{\text {loc }}^{1,2}(\mathbb{R})$ with $\left\|q^{\prime}\right\|_{L^{2}(\mathbb{R})} \leq C$ one has (letting $\phi(z):=$ $q(z+1)-q(z)) \phi(z) \leq C$ for all $z$ by (5), and hence (letting $\sup _{[-C, C]}\left|V^{\prime \prime}\right|=: C_{1}$ and
using $V(0)=V^{\prime}(0)=0$ ) using $V(0)=V^{\prime}(0)=0$ )

$$
V(\phi(z)) \leq \frac{C_{1}}{2} \phi(z)^{2} \leq \frac{C_{1}}{2} \int_{z}^{z+1}\left(q^{\prime}\right)^{2}
$$

Now recalling $V_{\jmath}^{k}=V\left(\phi_{\jmath}^{k}\right)\left(\phi_{\jmath}^{k}:=q_{\jmath}^{k}(z+1)-q_{j}^{k}(z)\right)$, dropping the superscript $k$ and using (11) we have

$$
\begin{aligned}
\int_{y_{k}-R_{1}-1}^{y_{k}-R_{1}} V_{1} & \leq \frac{C_{1}}{2} \int_{y_{k}-R_{1}-1}^{y_{k}-R_{1}}\left(\int_{z}^{z+1}\left(q_{1}^{\prime}\right)^{2}\right) d z \\
& =\frac{C_{1}}{2} \int_{y_{k}-R_{1}-1}^{y_{k}-R_{1}}\left(\int_{\max \left\{z, y_{k}-R_{1}\right\}}^{z+1}\left(q_{1}^{\prime}\right)^{2}\right) d z \\
& \leq \frac{C_{1}}{2} \int_{y_{k}-R_{1}}^{y_{k}+1}\left(q^{\prime}\right)^{2}
\end{aligned}
$$


and similarly

$$
\begin{gathered}
\int_{y_{k}+R_{1}-1}^{y_{k}+R_{1}} V_{1} \leq \frac{C_{1}}{2} \int_{y_{k}+R_{1}-1}^{y_{k}+R_{1}}\left(q^{\prime}\right)^{2}, \\
\int_{y_{k}-R_{2}-1}^{y_{k}-R_{2}} V_{2} \leq \frac{C_{1}}{2} \int_{y_{k}-R_{2}-1}^{y_{k}-R_{2}}\left(q^{\prime}\right)^{2}, \\
\int_{y_{k}+R_{2}}^{y_{k}+R_{2}-1} V_{2} \leq \frac{C_{1}}{2} \int_{y_{k}+R_{2}}^{y_{k}+1}\left(q^{\prime}\right)^{2} .
\end{gathered}
$$

Now since $\int_{\mathbb{R}}\left(q^{\prime}\right)^{2} \leq C^{2}$,

$$
\begin{array}{ll}
\min _{R_{1} \in\left[R, R+\frac{1}{3}\left(R^{k}-R\right)\right]_{\left[y_{k}-R_{1}-1, y_{k}-R_{1}\right] \cup\left[y_{k}+R_{1}-1, y_{k}+R_{1}\right]}} & V_{1} \leq \delta(k), \\
\min _{R_{2} \in\left[R+\frac{2}{3}\left(R^{k}-R\right), R^{k}\right]_{\left[y_{k}-R_{2}-1, y_{k}-R_{2}\right] \cup\left[y_{k}+R_{2}-1, y_{k}+R_{2}\right]}} V_{2} \leq \delta(k),
\end{array}
$$

where

$$
\delta(k)=\frac{C_{1} C^{2}}{2} \frac{1}{\frac{1}{3}\left(R^{k}-R\right)-1} \rightarrow 0 \quad \text { as } \quad k \rightarrow \infty .
$$

Choose $R_{1}, R_{2}$ so that the above minima are attained, then

$$
\begin{aligned}
\int_{\mathbb{R}}\left|V-V_{1}-V_{2}\right|= & \int_{\left[y_{k}-R_{1}-1, y_{k}-R_{1}\right] \cup\left[y_{k}+R_{1}-1, y_{k}+R_{1}\right]}\left|V-V_{1}\right| \\
& +\int_{\left[y_{k}-R_{2}-1, y_{k}-R_{2}\right] \cup\left[y_{k}+R_{2}-1, y_{k}+R_{2}\right]}\left|V-V_{2}\right| \\
& +\int_{\left[y_{k}-R_{2}, y_{k}-R_{1}-1\right] \cup\left[y_{k}+R_{1}, y_{k}+R_{2}-1\right]} V \\
\leq & 2 \delta(k)+\int_{B_{R^{k}+1}\left(y_{k}\right)} V-\int_{B_{R-1}\left(y_{k}\right)} V \\
& <2 \delta(k)+(\alpha+\varepsilon)-(\alpha-\varepsilon)=2 \delta(k)+2 \varepsilon .
\end{aligned}
$$

Also,

$$
\begin{aligned}
& \int_{\mathbb{R}} V_{1}=\int_{\left[y_{k}-R_{1}-1, y_{k}-R_{1}\right] \cup\left[y_{k}+R_{1}-1, y_{k}+R_{1}\right]} V_{1}+\int_{\left[y_{k}-R_{1}, y_{k}+R_{1}-1\right]} V, \\
& \int_{\mathbb{R}} V_{2} V_{\left[y_{k}-R_{2}-1, y_{k}-R_{2}\right] \cup\left[y_{k}+R_{2}-1, y_{k}+R_{2}\right]} V_{\mathbb{R} \backslash\left[y_{k}-R_{2}-1, y_{k}+R_{2}\right]} V,
\end{aligned}
$$


where the first term in both (12) and (13) is less or equal to $\delta(k)$ and the second term lies, respectively, in the interval

$$
\left[\int_{B_{R-1}\left(y_{k}\right)} V, \int_{B_{R^{k}+1}\left(y_{k}\right)} V\right] \subset(\alpha-\varepsilon, \alpha+\varepsilon)
$$

and in

$$
\left[K-\int_{B_{R^{k}+1}\left(y_{k}\right)} V, K-\int_{B_{R-1}\left(y_{k}\right)} V\right] \subset((K-\alpha)-\varepsilon,(K-\alpha)+\varepsilon),
$$

due to (9) and (10). Since $\delta(k) \rightarrow 0$ as $k \rightarrow \infty$, (iii) follows. The proof of the lemma is complete.

Our next aim is to investigate whether or not alternatives (ii) (vanishing) and (iii) (splitting) in Lemma 2 can occur if $\left\{q^{k}\right\}$ is a minimising sequence. A general strategy as to how to exclude splitting is presented in [3]: Up to minor technicalities, it is clear that here and in much more general cases, no minimising sequence splits if and only if the subadditivity inequalities (S) in Lemma 3a below hold true.

For our problem, it turns out that a similar strategy is possible concerning vanishing: No minimising sequence vanishes if and only if a certain bound on the minimum energy $((\mathrm{E})$ in Lemma $3 b$ below) is satisfied.

Lemma 3a (compare [3, Theorem II.1]). Let $V$ be as in Lemma 2, and let $K>0$ be fixed. Then the following two statements are equivalent:

(not iii) No minimising sequence $\left\{q^{k}\right\} \subset \mathscr{t}_{K}$ of $T$ splits (i.e. satisfies (iii) of Lemma 2)

$$
T_{K}<T_{\alpha}+T_{K-\alpha} \text { for all } \alpha \in(0, K) \text {. }
$$

Lemma 3b. Let $V$ be as in Lemma 1 , and let $K>0$ be fixed. Then the following three statements are equivalent:

(not ii) No minimising sequence $\left\{q^{k}\right\} \subset \mathscr{\ell}_{K}$ of $T$ vanishes (i.e. satisfies (ii) of Lemma l),

(not ii) There exists $\varepsilon(K)>0$ such that every minimising sequence $\left\{q^{k}\right\} \subset \cdot \mathscr{b}_{K}$ of $T$ satisfies the a-priori estimate

$$
\begin{gathered}
\liminf _{k \rightarrow \infty}\left\|q^{k}(\cdot+1)-q^{k}(\cdot)\right\|_{L^{\infty}(\mathbb{R})}>\varepsilon, \\
V^{\prime \prime}(0) \cdot T_{K}<K .
\end{gathered}
$$

Proof of Lemma $3 a$. (S) $\Rightarrow$ (not iii): Suppose there was a minimising sequence $\left\{q^{k}\right\} \subset$ $\mathscr{C}_{K}$ satisfying (iii) for some $\alpha \in(0, K)$. Then defining $\alpha_{k}:=U\left(q_{1}^{k}\right)$ and $\beta_{k}:=U\left(q_{2}^{k}\right)$, letting $k \rightarrow \infty$ and using the continuity of $T_{K}$ (Lemma $1(\mathrm{~d})$ ) one would obtain

$$
T_{K} \geq \liminf _{k \rightarrow \infty}\left(T\left(q_{1}^{k}\right)+T\left(q_{2}^{k}\right)\right) \geq \liminf _{k \rightarrow \infty}\left(T_{\alpha_{k}}+T_{\beta_{k}}\right)=T_{\alpha}+T_{K-\alpha},
$$

contradicting $(S)$.

(not iii) $\Rightarrow(\mathrm{S})$ : Assume (S) does not hold, i.e. $\exists \alpha \in(0, K)$ such that $T_{K} \geq$ $T_{\alpha}+T_{K-\alpha}$; it suffices to construct a minimising sequence in $\mathscr{b}_{K}$ satisfying (iii). This is easy: Pick minimising sequences $q_{\alpha}^{k} \subset ._{t_{\alpha}}, q_{K-\alpha}^{k} \subset \mathscr{A}_{K-\alpha}$ of $T$; by arguing similarly to the proof of the continuity of $T_{K}$ (Lemma $1(\mathrm{~d})$ ), we may assume without loss that the support of the derivatives $\left(q_{\alpha}^{k}\right)^{\prime},\left(q_{\kappa-\alpha}^{k}\right)^{\prime}$ is contained in some balls $B_{R_{k}}(0)$. Then the sequence $q^{k}(z):=q_{K-\alpha}^{k}\left(z+R_{k}+k\right)+q_{K-\alpha}^{k}\left(z-R_{k}-k\right)+C_{k}$ $\left(C_{k}\right.$ chosen so that $\left.q^{k}(0)=0\right)$ has the required properties. 
Proof of Lemma $3 b$. This somewhat mysterious looking statement relies on the fact that it is possible to explicitly calculate the limit $\varepsilon \searrow 0$ of the infima

$$
T_{K, \varepsilon}:=\inf \left\{T(q): q \in \mathscr{b}_{K},\|q(\cdot+1)-q(\cdot)\|_{L^{\infty}(\mathbb{R})} \leq \varepsilon\right\} .
$$

(Note that the above subsets of $\mathscr{A}_{K}$ are nonempty and hence the $T_{K, \varepsilon}$ well-defined, because for small enough $\varepsilon>0$ and appropriately chosen $L(\varepsilon), q_{\varepsilon, L}$ lies in the above set.) Namely, we shall show

$$
T_{K, \varepsilon} \rightarrow \frac{K}{V^{\prime \prime}(0)} \quad \text { as } \quad \varepsilon \rightarrow 0
$$

where the limit is to be understood as $+\infty$ in case $V^{\prime \prime}(0)=0$. To derive this formula, we first extimate $\liminf _{\varepsilon \rightarrow 0} T_{K, \varepsilon}$ from below, as follows: Let $\left\{q^{k}\right\} \subset \mathscr{A}_{K}$ be an arbitrary sequence such that $\left\|\phi^{k}\right\|_{L^{\infty}(\mathbb{R})} \rightarrow 0$ (where $\phi^{k}=q^{k}(\cdot+1)-q^{k}(\cdot)$ as usual), then by (6),

$$
\begin{aligned}
K & =\int_{\mathbb{R}} V\left(\phi^{k}\right) \leq \frac{1}{2} \sup _{\left[-\left\|\phi^{k}\right\|_{\infty},\left\|\phi^{k}\right\|_{\infty}\right]}\left|V^{\prime \prime}\right| \cdot \int_{\mathbb{R}} \int_{z}^{z+1}\left(\left(q^{k}\right)^{\prime}\right)^{2} \\
& =\sup _{\left[-\left\|\phi^{k}\right\|_{\infty},\left\|\phi^{k}\right\|_{\infty}\right]}\left|V^{\prime \prime}\right| \cdot T\left(q^{k}\right),
\end{aligned}
$$

thus $K \leq V^{\prime \prime}(0) \liminf _{k \rightarrow \infty} T\left(q^{k}\right)$ and in particular

$$
K \leq V^{\prime \prime}(0) \liminf _{k \rightarrow \infty} T_{K, \varepsilon} .
$$

This estimate is so good that in the limit $\varepsilon \rightarrow 0$ it becomes optimal. To show this, consider again the functions $q_{\Lambda, L}$ defined in (8). Recall that for $L \geq 1$, $T\left(q_{\Lambda, L}\right)=\frac{1}{2} \Lambda^{2} L$ and $U\left(q_{\Lambda, L}\right)=(L-1) V(\Lambda)+2 \int_{0}^{1} V(\Lambda z) d z$. Pick $\Lambda_{0}$ so small that $U\left(q_{\Lambda, 1}\right)<K$ for all $\Lambda \in\left(0, \Lambda_{0}\right)$, then for every such $\Lambda$ there exists $L(\Lambda) \geq 1$ such that $U\left(q_{\Lambda, L}\right)=K$. Now

$$
\frac{(L-1) V(\Lambda)}{\frac{1}{2} \Lambda^{2} L} \leq \frac{K}{T\left(q_{\Lambda, L(\Lambda)}\right)} \leq \frac{(L-1) V(\Lambda)+2 \int_{0}^{1} V(\Lambda z) d z}{\frac{1}{2} \Lambda^{2} L} .
$$

Since $V(\Lambda)=\frac{1}{2} V^{\prime \prime}(0) \Lambda^{2}+o\left(\Lambda^{2}\right)$ and $L(\Lambda) \rightarrow \infty$ as $\Lambda \rightarrow 0$, both the left-hand side and the right-hand side in (16) converge to $V^{\prime \prime}(0)$ as $\Lambda \rightarrow 0$, consequently

$$
K=V^{\prime \prime}(0) \lim _{\Lambda \rightarrow 0} T\left(q_{\Lambda, L(\Lambda)}\right) \geq V^{\prime \prime}(0) \limsup _{\varepsilon \rightarrow 0} T_{K, \varepsilon} .
$$

This establishes (14), and hence proves (not ii) ${ }^{\sim} \Leftrightarrow(\mathrm{E})$. It remains to prove (not ii) $\Leftrightarrow$ (not ii) $\sim$. " $\Rightarrow$ " is trivial, and to demonstrate " $\Leftarrow$ " it suffices to show that given $C, \varepsilon>0$ there exist $\varepsilon_{1}(\varepsilon, C), R(\varepsilon, C)>0$ such that all $q \in X$ with $\|q\| \leq C$, $\|\phi\|_{L^{\infty}(\mathbb{R})} \geq \varepsilon$ satisfy

$$
\sup _{y \in \mathbb{R}} \int_{B_{R}(y)} V(\phi)>\varepsilon_{1}
$$


Pick $y$ such that $V(\phi(y)) \geq \varepsilon$. By (5), $\|\phi\|_{L^{\infty}(\mathbb{R})} \leq C$, and (letting $\left.C_{0}:=\sup _{[-C, C]}\left|V^{\prime}\right|\right)$
we compute for $z \geq y$

$$
\begin{aligned}
|V(\phi(z))-V(\phi(y))| & \leq C_{0}|\phi(z)-\phi(y)| \leq C_{0} \int_{y}^{z}\left(\left|q^{\prime}(\cdot+1)\right|+\left|q^{\prime}(\cdot)\right|\right) \\
& \leq 2 C C_{0} \sqrt{z-y}
\end{aligned}
$$

and hence

$$
V(\phi(y)) \geq \varepsilon-2 C C_{0} \sqrt{z-y},
$$

so that (17) holds with $r=\left(\frac{\varepsilon}{2 C C_{0}}\right)^{2}, \varepsilon_{1}=\int_{0}^{R}\left(\varepsilon-2 C C_{0} \sqrt{z}\right) d z$. This completes the
proof of the lemma.

As a reward for all the technicalities above, we are now able to prove the following:

Proposition 1. Let $V$ be as in Lemma 1, and let $K>0$ be fixed. Assume furthermore that the subadditivity inequalities (S) and the energy inequality (E) hold. Then T attains its infimum on $t_{K}$.

Proof. This is an immediate consequence of Lemmas 2, 3a, and 3b, and of standard arguments in the calculus of variations. Take a minimising sequence $\left\{q^{k}\right\} \subset \mathscr{A}_{K}$. By Lemmas 2, 3a, and 3b, after passing to a subsequence we may assume that $\left\{q^{k}\right\}$ satisfies the compactness property (i) of Lemma 2. By the invariance of $T$ and $U$ under translations and under adding constants, we can assume that the $y_{k}$ are all zero, otherwise replace $q^{k}$ by $q^{k}\left(y_{k}+\cdot\right)-q^{k}\left(y_{k}\right)$. Clearly, $\left\{q^{k}\right\}$ is bounded in $X$, so we can choose a weakly convergent subsequence $q^{k} \longrightarrow q \in X$. By weak lower semicontinuity of the norm in $X, T(q) \leq \inf _{K} T$. It remains to show that $U(q)=K$ (survival of the constraint). By (i) and the fact that the $y_{k}$ are all zero, all we have to prove is

$$
\int_{B_{R}(0)} V\left(\phi^{k}\right) \rightarrow \int_{B_{R}(0)} V(\phi) \quad \text { as } \quad k \rightarrow \infty \quad \forall R>0,
$$

but this is immediate from the compact Sobolev embedding $W^{1,2}\left(B_{R}(0)\right) \hookrightarrow$ $L^{\infty}\left(B_{R}(0)\right)$ which implies $q^{k} \rightarrow q$ strongly in $L^{\infty}\left(B_{R}(0)\right)$.

Note that so far none of our analysis has involved imposing any "constitutive assumptions" on the interaction potential $V$ (other than the assumption made at the very beginning that 0 is a local minimum so that the state $q_{n}=0$ is a stable equilibrium state of the Hamiltonian system (1)). We now investigate for which potentials $V$ the conditions (E) and (S) hold, and it turns out that the answer depends in an interesting way on the growth behaviour of $V$.

Proposition 2. Let $V$ be as in Lemma 1, in addition $V$ superquadratic (i.e. $\frac{V(r)}{r^{2}}$ strictly increasing with $|r|)$. Then there exists $K_{0} \geq 0$ such that for $K>K_{0}$, (E) and (S) hold; in particular (by Proposition 1) $T$ attains its infimum on $t_{K}$ for all $K>K_{0}$. In contrast, if $V$ is subquadratic (i.e. $\frac{V(r)}{r^{2}}$ nonincreasing with $\left.|r|\right)$ then for every $K>0$ neither $(\mathrm{E})$ nor $(\mathrm{S})$ hold, and the infimum of $T$ on $\mathscr{t}_{K}$ is not attained. 
Proof. First, for the sake of motivation let us look at exactly quadratic potentials $V$. In this case, since $V^{\prime \prime} \equiv$ const, (15) and (14) give $T_{K}$ explicitly as $T_{K}=\frac{K}{V^{\prime \prime}(0)}$ for all $K \geq 0$, and in particular $T_{K}=T_{\alpha}+T_{K-\alpha}$ for all $\alpha \in[0, K]$. Hence instead of the strict inequalities $(\mathrm{E})$ and $(\mathrm{S})$ we have equality.

Now let us deal with the superquadratic case. To begin with, we have

$$
V(r)>\frac{1}{2} V^{\prime \prime}(0) r^{2}
$$

for all $r \neq 0$, since $\frac{V(r)}{r^{2}}$ increases strictly with $|r|$ and tends to $\frac{1}{2} V^{\prime \prime}(0)$ as $r \rightarrow 0$. Now fix $\Lambda>0$ and recall the functions $q_{\Lambda, L}$ defined in (8). To prove (E), it is enough to find $q_{\Lambda, L} \in \mathscr{b}_{K}$ such that

$$
\begin{aligned}
U\left(q_{\Lambda, L}\right) & >V^{\prime \prime}(0) T\left(q_{\Lambda, L}\right) \\
\Leftarrow(L-1) V(\Lambda) & >\frac{1}{2} V^{\prime \prime}(0) \Lambda^{2} L .
\end{aligned}
$$

Dividing by $L$, letting $L \rightarrow \infty$ and using (19), we see that (20) holds for $L \geq$ some $L_{0}$, hence (E) holds for $K \geq K_{0}:=U\left(q_{\Lambda, L}\right)$.

Remark 1. If $V^{\prime \prime}(0)=0$, then the above argument shows that (E) in fact holds for all $K>0$. If $V^{\prime \prime}(0)$ is non-zero, the question as to whether (E) continues to hold for small $K$ is more delicate and will be addressed after the proof of the proposition has been completed.

We claim that with this definition of $K_{0}$, (S) holds for $K \geq 2 K_{0}$, and formulate this as a separate lemma:

Lemma 4. Let $V$ be as in Lemma 1 and superquadratic, and assume (E) holds for $K>K_{0}$. Then (S) holds for $K>2 K_{0}$.

Proof. As pointed out in [3] (Lemma II.1), it suffices to verify the somewhat simpler inequality $T_{\theta \alpha}<\theta T_{\alpha}$ for all $\alpha \in(0, K)$ and all $\theta \in\left(1, \frac{K}{\alpha}\right]$; in fact strict inequality is only needed for $\alpha \geq \frac{K}{2}$ :

Lemma 5. Let $h:[0, K] \rightarrow \mathbb{R}$, and assume

$$
\left\{\begin{array}{l}
h(\theta \alpha) \leq \theta h(\alpha) \forall \alpha \in\left(0, \frac{K}{2}\right), \forall \theta \in\left(1, \frac{K}{\alpha}\right] \\
h(\theta \alpha)<\theta h(\alpha) \forall \alpha \in\left[\frac{K}{2}, K\right), \quad \forall \theta \in\left(1, \frac{K}{\alpha}\right] .
\end{array}\right.
$$

Then $h(K)<h(\alpha)+h(K-\alpha)$ for all $\alpha \in(0, K)$.

Proof of Lemma 5. This is elementary: Assume without loss of generality $\alpha \geq \frac{K}{2}$, then $h(K)<\frac{K}{\alpha} h(\alpha)$ and $h(K) \leq \frac{K}{K-\alpha} h(K-\alpha)$, hence $h(\alpha)+h(K-\alpha)>$ $\left(\frac{\alpha}{K}+\frac{K-\alpha}{K}\right) h(K)$.

Hence in order to prove Lemma 4, we only need to show that for $h(\alpha)=T_{\alpha}$, ( $\tilde{\mathrm{S}})$ holds for all $K>2 K_{0}$. This is the consequence of a (simple) scaling argument, 
together with the (nontrivial) fact that for $q$ near the minimum of $T$, the higherthan-quadratic terms of $V$ really contribute to $U(q)$, since by (E) and Lemma $3 \mathrm{~b}$ the maximum $\|\phi\|_{\infty}$ is bounded away from zero. So assume $K \geq 2 K_{0}$ and fix $\alpha \in(0, K)$, $\theta \in\left(1, \frac{K}{\alpha}\right]$. We only deal with the case $\alpha \geq \frac{K}{2}$, the other case being easier. By (E), Lemma $3 \mathrm{~b}$, and the boundedness of minimising sequences, $\exists \varepsilon, C>0$ such that $T_{\alpha}=\inf _{\ell_{\alpha, \varepsilon, C}} T$, where

$$
\mathscr{C}_{\alpha, \varepsilon, C}:=\left\{q \in X: U(q)=\alpha,\|\phi\|_{\infty} \geq \varepsilon,\|q\| \leq C\right\} .
$$

By (18), there exists $\alpha_{0}>0$ such that

$$
\int_{\left\{z \in \mathbb{R}:|\phi(z)| \geq \frac{\varepsilon}{2}\right\}} V(\phi(z)) \geq \alpha_{0} \quad \forall q \in \mathscr{A}_{\alpha, \varepsilon, C} .
$$

Also, let

$$
\theta_{0}:=\min _{\substack{|r| \in\left[\frac{\varepsilon}{2}, C\right] \\ \lambda \in[1, \sqrt{\theta}]}} \frac{V(\lambda r)}{\lambda^{2} V(r)}
$$

and note $\theta_{0}>1$ (by the superquadraticity of $V$ ). Now take $q \in \mathscr{A}_{\alpha, \varepsilon, C}$. Since $U(\lambda q)=\alpha$ at $\lambda=1$ and $U(\lambda q)=U(\sqrt{\theta} q) \geq \theta U(q)=\theta \alpha$ at $\lambda=\sqrt{\theta}$, there exists $\lambda(\theta, q) \in[1, \sqrt{\theta}]$ such that $U(\lambda(\theta, q), q)=\theta \alpha$. We show that $\lambda(\theta, q)$ is in fact strictly smaller than $\sqrt{\theta}$ :

$$
\begin{gathered}
\theta \alpha=U(\lambda q)=\int_{\left\{z \in \mathbb{R}:|\phi(z)|<\frac{\varepsilon}{2}\right\}} V(\lambda \phi)+\int_{\left\{z \in \mathbb{R}:|\phi(z)| \geq \frac{\varepsilon}{2}\right\}} V(\lambda \phi) \\
\geq \lambda^{2} \int_{\left\{z \in \mathbb{R}:|\phi(z)|<\frac{\varepsilon}{2}\right\}} V(\phi)+\theta_{0} \lambda^{2} \int_{\left\{z \in \mathbb{R}:|\phi(z)| \geq \frac{\varepsilon}{2}\right\}} V(\phi) \\
\geq \lambda^{2}\left(\left(\alpha-\alpha_{0}\right)+\theta_{0} \alpha_{0}\right) \alpha,
\end{gathered}
$$

i.e. $\lambda \leq \lambda_{0}$ where

$$
\lambda_{0}=\left(\frac{\theta}{\left(\alpha-\alpha_{0}\right)+\theta_{0} \alpha_{0}}\right)^{1 / 2}
$$

Consequently

$$
T_{\theta \alpha} \leq \inf _{q \in \mathscr{Z}_{\alpha, \varepsilon, C}} T(\lambda(\theta, q) q) \leq \lambda_{0}^{2} \inf _{q \in \ell_{\alpha, \varepsilon, C}} T(q)=\frac{\theta}{\left(\alpha-\alpha_{0}\right)+\theta_{0} \alpha_{0}} T_{\alpha},
$$

proving $(\tilde{\mathrm{S}})$. The proof of Lemma 4 , and thus of the first part of the proposition, is complete.

Finally, we deal with the case of subquadratic potentials $V$. Fix $K>0$. To show nonattainment, in view of (14) it suffices to show that

$$
U(q)<V^{\prime \prime}(0) T(q) \quad \forall q \in \mathscr{A}_{K} .
$$


This follows from a slight refinement of the estimate (15) used in the proof of Lemma 3b. First of all, analogously to (19) we have $V(r) \leq \frac{1}{2} V^{\prime \prime}(0) r^{2} \forall r \in \mathbb{R}$. Also, we use the fact that in the Schwarz inequality $\int_{a}^{b} f g \leq\|f\|_{L^{2}(a, b)}\|g\|_{L^{2}(a, b)}$, equality occurs if and only if one function is a multiple of the other. Now since $q \in A_{K}$ and $K>0, \int_{\mathbb{R}}\left(q^{\prime}\right)^{2}<\infty$ and $q^{\prime} \not \equiv 0$, thus there exists $z \in \mathbb{R}$ such that $q^{\prime} \not \equiv$ const on the interval $(z, z+1)$, hence

$$
U(q) \leq \frac{1}{2} V^{\prime \prime}(0) \int_{\mathbb{R}}\left(\int_{z}^{z+1} q^{\prime}\right)^{2}<\frac{1}{2} V^{\prime \prime}(0) \int_{\mathbb{R}} \int_{z}^{z+1}\left(q^{\prime}\right)^{2}=V^{\prime \prime}(0) T(q) .
$$

proving (21). Hence the infimum of $T$ is not attained. Also, (21) clearly contradicts (E), and finally (S) cannot hold since (14) and (21) show $T_{K}=\frac{K}{V^{\prime \prime}(0)}$ for all $K$. The
proof of the proposition is complete.

In the last part of this section, we investigate the question of attainment in the case left open by Proposition 2, namely superquadratic potentials $V$ (with nonvanishing second derivative at zero) but low energies $K$. In this case it is more difficult to establish existence than in the high energy range, since - as remarked in Sect. 2. - the smaller the prescribed potential energy, the "closer" we are to a situation where the minimum is not attained. Precisely, combining (13) and Lemma 1(a), $K \leq T_{K} \cdot \quad \sup \left|V^{\prime \prime}\right|$, hence by Lemma $1(\mathrm{~d})$,

$$
\frac{T_{K}-T_{K, 0}}{K} \rightarrow 0 \quad(K \rightarrow 0)
$$

(where $T_{K, 0}:=\lim _{\varepsilon \rightarrow 0} T_{K, \varepsilon}=K / V^{\prime \prime}(0)$ ). This implies that if we want to find $q \in \mathscr{A}_{K}$ with $T(q)<T_{K, 0}$, then the allowable error by which the energy of its normalised shape function $q /\|\phi\|_{L^{2}}$ (normalised so that the difference function $\phi /\|\phi\|_{L^{2}}$ has $L^{2}$-norm one) may differ from the energy of the normalized shape function of an exact minimiser $q_{0}$ becomes arbitrarily small as $K \rightarrow 0$, because for such $q, T\left(q /\|\phi\|_{L^{2}}\right)-T\left(q_{0} /\left\|\phi_{0}\right\|_{L^{2}}\right) \rightarrow 0$ [by (22)].

The result below grew out of the effort to deal with the (symmetrised) model potential $V(\phi)=\frac{1}{2} \phi^{2}+\frac{1}{3} a|\phi|^{3}$, whose continuum limit (the Boussinesq equation) possesses exact travelling waves of the form $q(z)=\Lambda \tanh (\beta z)$, so that for low energies the waves on the lattice whose existence we want to establish can be expected to be well-approximated by these functions. It turns out that these "guesses" are good enough to establish (E) for a larger class of potentials:

Proposition 3. Let $V$ be as in Lemma $1, V$ superquadratic (recall that then automatically $V(r)>\frac{1}{2} V^{\prime \prime}(0) r^{2} \forall r \neq 0$ ), in addition $V(\cdot)$ superquadratic with a sufficiently large algebraic rate in some neighbourhood of zero, i.e.

$$
V(r)=\frac{1}{2} V^{\prime \prime}(0) r^{2}+\varepsilon|r|^{p}+o\left(r^{p}\right) \text { as } r \rightarrow 0, \text { for some } \varepsilon>0 \text { and some } 2<p<6 .
$$

Then (E) and (S) hold for all $K>0$; in particular (by Proposition 1), $T$ attains its infimum on $A_{K}$ for all $K>0$. 
Proof. In view of Lemma 4, it suffices to prove (E). As motivated above, we will use the functions $q_{\Lambda, \beta}(z)=\Lambda \tanh (\beta z)$. First we shall show that given $K>0$, there is a $\Lambda(\beta)$ such that $U\left(q_{\Lambda, \beta}\right)=K$; and subsequently that the inequality $V^{\prime \prime}(0) T\left(q_{\Lambda, \beta}\right)<U\left(q_{\Lambda, \beta}\right)$ holds. We perform this by expanding $U\left(q_{\Lambda, \beta}\right)$ and $T\left(q_{\Lambda, \beta}\right)$ for small $\beta$ as a power series in $\beta$. Clearly $q_{\Lambda, \beta}(z) \in X$, and

$$
\phi_{\Lambda, \beta}:=q_{\Lambda, \beta}\left(z+\frac{1}{2}\right)-q_{\Lambda, \beta}\left(z-\frac{1}{2}\right)=\frac{2 \Lambda \tanh \left(\frac{1}{2} \beta\right) \operatorname{sech}^{2}(\beta z)}{1-\tanh ^{2}(\beta z) \tanh ^{2}\left(\frac{1}{2} \beta\right)} .
$$

We expand $\phi_{\Lambda, \beta}$ as a power series using: $2 \tanh (\beta / 2) \sim \beta-\frac{1}{12} \beta^{3}+O\left(\beta^{5}\right)$, and $\frac{1}{1-x} \sim 1+x+O\left(x^{2}\right)$. Hence

$$
\begin{aligned}
\phi & =\Lambda\left[\beta-\frac{1}{12} \beta^{3}+O\left(\beta^{5}\right)\right]\left[1+\frac{1}{4} \beta^{2} \tanh ^{2}(\beta z)+O\left(\beta^{4}\right)\right] \operatorname{sech}^{2}(\beta z) \\
& =\Lambda\left[\beta+\frac{1}{4} \beta^{3} \tanh ^{2}(\beta z)-\frac{1}{12} \beta^{3}+O\left(\beta^{5}\right)\right] \operatorname{sech}^{2}(\beta z),
\end{aligned}
$$

where here and below the $o, O$ notation refers to behaviour as $\beta \rightarrow 0$. It is straightforward to calculate $q_{\Lambda, \beta}^{\prime}(z)=\Lambda \beta \operatorname{sech}^{2}(\beta z)$, hence

$$
T\left(q_{\Lambda, \beta}\right)=\frac{1}{2} \Lambda^{2} \beta \int_{\mathbb{R}} \operatorname{sech}^{4}(x) d x .
$$

Calculation of $U(q)$ requires a little more effort. Before we can expand $V(\phi)$, we need to estimate the term $\beta \Lambda(\beta) \approx \sup |\phi(z)|$ to show that the $o\left(|r|^{p}\right)=o\left(\|\phi\|_{\infty}^{p}\right)$ term can be ignored (i.e. $\beta \Lambda(\beta) \sim O(1))^{z}$.

By (19), $\Lambda(\beta)$ will be smaller than the solution $\Lambda_{0}(\beta)$ of the equation

$$
\int_{\mathbb{R}} \frac{1}{2} V^{\prime \prime}(0) \phi_{\Lambda_{0}, \beta}^{2}=K \text {. }
$$

The left-hand side of this simplifies to: $\frac{1}{2} V^{\prime \prime}(0) \Lambda_{0}^{2}\left[\beta^{2}+O\left(\beta^{4}\right)\right] \int_{\mathbb{R}} \operatorname{sech}^{4}(\beta z) d z=$ $\frac{1}{2} V^{\prime \prime}(0) \Lambda_{0}^{2} \beta\left[1+O\left(\beta^{2}\right)\right] \int_{\mathbb{R}} \operatorname{sech}^{4} x d x$. Hence

$$
\underbrace{\Lambda_{0}^{2} \beta}_{r(\beta)}\left[1+O\left(\beta^{2}\right)\right] \underbrace{\frac{1}{2} V^{\prime \prime}(0) \int_{\mathbb{R}} \operatorname{sech}^{4} x d x}_{C_{1}}=K,
$$

$$
\begin{gathered}
\text { and } r(\beta)=\frac{K}{C_{1}\left[1+O\left(\beta^{2}\right)\right]}=\frac{K}{C_{1}}\left[1+O\left(\beta^{2}\right)\right] ; \Lambda_{0}(\beta)=\sqrt{\frac{r(\beta)}{\beta}}=\sqrt{\frac{K}{C_{1} \beta}}+O\left(\beta^{1 / 2}\right) ; \\
\beta \Lambda(\beta) \leq \beta \Lambda_{0}=O\left(\beta^{1 / 2}\right) .
\end{gathered}
$$

We are now in a position to expand $U\left(q_{\Lambda, \beta}\right)$, knowing the order of magnitude of $\Lambda$ is at most $\beta^{-1 / 2}$. Firstly we expand $V(\phi)$,

$$
\begin{aligned}
V(\phi)= & \frac{1}{2} V^{\prime \prime}(0) \phi^{2}+\varepsilon|\phi|^{p}+o\left(|\phi|^{p}\right) \\
= & \frac{1}{2} \Lambda^{2} V^{\prime \prime}(0)\left[\beta^{2}+\frac{1}{2} \beta^{4} \tanh ^{2}(\beta z)-\frac{1}{6} \beta^{4}+O\left(\beta^{6}\right)\right] \operatorname{sech}^{2}(\beta z) \\
& +\varepsilon \Lambda^{p}\left[\beta^{p}+o\left(\beta^{p}\right)\right] \operatorname{sech}^{2}(\beta z)+o\left(\Lambda^{p} \beta^{p} \operatorname{sech}^{2 p}(\beta z)\right) .
\end{aligned}
$$


Hence the expression for $U\left(q_{\Lambda, \beta}\right)$,

$$
\begin{aligned}
U\left(q_{\Lambda, \beta}\right)= & \frac{1}{2} \Lambda^{2} \beta V^{\prime \prime}(0) \\
& \times[\int_{\mathbb{R}} \operatorname{sech}^{4}(x) d x-\beta^{2} \underbrace{\int_{\mathbb{R}}\left(\frac{1}{2} \tanh ^{2} x-\frac{1}{6}\right) \operatorname{sech}^{4}(x) d x}_{C_{2}}+O\left(\beta^{4}\right)] \\
& +\varepsilon \Lambda^{p} \beta^{p-1} \int_{\mathbb{R}} \operatorname{sech}^{2 p}(x) d x+o\left(\beta^{p / 2-1}\right) .
\end{aligned}
$$

Thus we have an asymptotic expression $\Lambda(\beta)=\sqrt{K / C_{1} \beta}+o\left(\beta^{-1 / 2}\right)$.

Finally, we need to check that the inequality $V^{\prime \prime}(0) T\left(q_{\Lambda, \beta}\right)<U\left(q_{\Lambda, \beta}\right)$ holds. The kinetic term is exactly equal to the highest order $\left(O\left(\Lambda^{2} \beta\right)\right)$ potential term, so the highest order terms cancel and we are left with

$$
\begin{aligned}
& U\left(q_{\Lambda, \beta}\right)-V^{\prime \prime}(0) T\left(q_{\Lambda, \beta}\right) \\
& \quad=\frac{1}{2} \Lambda^{2} \beta^{3} V^{\prime \prime}(0)\left[C_{2}+O\left(\beta^{2}\right)\right]+\varepsilon \Lambda^{p} \beta^{p-1} \int_{\mathbb{R}} \operatorname{sech}^{2 p}(x) d x+o\left(\beta^{p / 2-1}\right) \\
& \quad=g_{1} \beta^{2}+o\left(\beta^{2}\right)+g_{2} \beta^{p / 2-1}+o\left(\beta^{p / 2-1}\right),
\end{aligned}
$$

where $g_{1}, g_{2}$ are constants (depending on $K, C_{1}, C_{2}$ but independent of $\beta, \Lambda$ ), and $g_{2}>0$. (Without calculation we know $g_{1} \leq 0$ because otherwise, for the exactly quadratic potential the inequality would hold for small $\beta$, contradicting Proposition 2 . In fact, $g_{1}<0$ since one calculates $C_{2}=-\frac{4}{45}$.) Hence provided $p / 2-1<2$, the inequality is valid for sufficiently small $\beta$. The condition forces $p<6$, as bound which could be raised if a sufficiently accurate estimate for the waveform can be found, e.g. by using the continuum limit of the lattice for the potential $V(r)=\frac{1}{2} V^{\prime \prime}(0) r^{2}+\varepsilon r^{p}$. However, for physically relevant potentials the results derived above are sufficient. It is feasible that the cubic coefficient of its Taylor expansion at zero would vanish due to symmetry arguments, but the quartic would then generically be non-zero.

\section{Are the Minimisers Travelling or Standing Waves?}

In this section we show that the minimisers constructed in Propositions 2 and 3 are indeed solutions to the travelling wave equation (3). While it is - up to fine points of rigour - clear that minimisers are solutions to the Euler-Lagrange equation

$$
q^{\prime \prime}(z)-\lambda\left(V^{\prime}(q(z+1)-q(z))-V^{\prime}(q(z)-q(z-1))\right)=0
$$

(for some Lagrange multiplier $\lambda \in \mathbb{R}$ ), the issue here is to determine the sign of $\lambda$. Note that only solutions of (23) with $\lambda>0$ give rise to a travelling wave on the lattice, otherwise (i.e. if $\lambda<0$ ) one would instead obtain a "standing wave."

Lemma 6. Let $V$ be as in Lemma 1, in addition $V$ increasing (decreasing) for $r>0$ $(r<0)$. Let $K>0$, and let $q \in \mathscr{A}_{K}$ be a minimiser of $T$ on $\mathscr{C}_{K}$. Then $q \in C^{2}(\mathbb{R})$, and $q$ satisfies (23) for some $\lambda>0$. In particular, $q$ solves the travelling wave equation (2) with $c=\lambda^{-1 / 2}$. 
Proof. Let $q \in \mathscr{C}_{K}$ be a minimiser of $T$. Clearly, $q$ also minimises $T$ on the larger set $\mathscr{\mathscr { S }}_{K}:=\{q \in Y: U(q)=K\}$, where $Y:=\left\{q \in W_{\text {loc }}^{1,2}(\mathbb{R}):\left\|q^{\prime}\right\|_{L^{2}(\mathbb{R})}<\infty\right\}$ (without the constraint $q(0)=0$ ). It is standard to check that $U$ - despite being an integral over an infinite domain - is Gateaux-differentiable as a functional from $Y$ to $\mathbb{R}$, and that its derivative is the functional obtained by differentiating the integrand. Indeed, letting $q, \zeta \in Y$, denoting the associated difference functions by

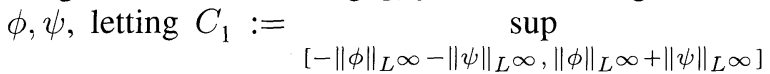

proof of Lemma 1(a), for all $|\varepsilon| \leq 1$

$$
\left|\frac{V(\phi+\varepsilon \psi)-V(\phi)}{\varepsilon}\right| \leq C_{1}(|\phi|+|\psi|)|\psi| \in L^{1}(\mathbb{R}),
$$

hence by dominated convergence $\lim _{\varepsilon \rightarrow 0} \frac{U(q+\varepsilon \zeta)-U(q)}{\varepsilon}$ exists and equals $\int_{\mathbb{R}} V^{\prime}(\phi) \psi$. Now consider the maps $f(\varepsilon, t):=T(q+\varepsilon \zeta+t q), g(\varepsilon, t):=U(q+\varepsilon \zeta+t q)$. Then $g$ satisfies the transversality condition $\nabla g(0,0) \neq 0\left(\right.$ since $\frac{\partial g}{\partial t}(0,0)=\int_{\mathbb{R}} V^{\prime}(\phi) \phi$ and $V^{\prime}(r) r>0$ for all $r \neq 0$ by hypothesis), hence by the Lagrange multiplier rule there
exists $\lambda \in \mathbb{R}$ such that

$$
0=\nabla f(0,0)-\lambda \nabla g(0,0)=\left(\begin{array}{l}
\int\left(q^{\prime} \zeta^{\prime}-\lambda V^{\prime}(\phi) \psi\right) \\
\int_{\mathbb{R}}\left(\left(q^{\prime}\right)^{2}-\lambda V^{\prime}(\phi) \phi\right)
\end{array}\right) .
$$

The second component shows $\lambda$ independent of $\zeta$ and $\lambda>0$, and (since $\zeta \in Y$ was arbitrary) the first component states that $q$ is a weak solution of (23). By the Sobolev embedding $W_{\text {loc }}^{1,2}(\mathbb{R}) \hookrightarrow C^{0}(\mathbb{R}), q \in C^{0}$, thus the nonlocal term $V^{\prime}(\phi)=V^{\prime}(q(\cdot+1)-q(\cdot)) \in C^{0}$, hence by (24) $q \in C^{2}$, and $q$ is a classical solution of (23). (Clearly, provided $V \in C^{\infty}$, we can iterate the argument, giving $q \in C^{\infty}$.) The proof of the lemma is complete.

\section{Monotonicity of the Minimisers}

Here we show that for symmetric interaction potentials $V$, the minimisers constructed in Propositions 2 and 3 must be monotone functions. Due to the nonlocal nature of the constraint subject to which one minimises, this is not immediately obvious. In fact numerical simulations and asymptotic analysis suggest that if the Hamiltonian (1) is changed to incorporate next-nearest neighbour interactions as well, monotonicity breaks down.

Lemma 7. Let $V$ be as in Lemma 1, in addition $V$ symmetric (i.e. $V(r)=V(-r)$ ) and superquadratic. Let $K>0$, and assume $q \in \mathscr{t}_{K}$ is a minimiser of $T$ on $\mathscr{A}_{K}$. Then $q$ is a monotone function; in particular its difference function $\phi$ is either $\geq 0$ or $\leq 0$.

Proof. Take a minimiser $q \in$ A $_{K}$, and consider the function

$$
\tilde{q}(z):=\int_{0}^{z}\left|q^{\prime}\right|
$$




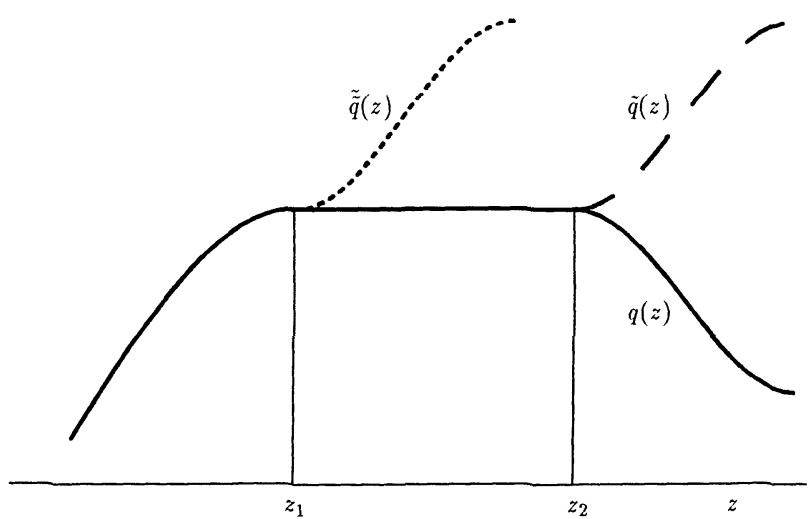

Fig. 2. Hypothetical shape of $q$, and the functions $\tilde{q}, \tilde{q}$ used in the Proof of Lemma 10

Denoting the corresponding difference function by $\tilde{\phi}$, we claim first of all that

$$
\tilde{\phi}(z)=|\phi(z)| \text { for all } z \in \mathbb{R} .
$$

" $\geq$ " is clear. Now suppose for contradiction that $\tilde{\phi}\left(z_{0}\right)>\left|\phi\left(z_{0}\right)\right|$ at some $z_{0}$. Then by the continuity of $\phi$ and $\tilde{\phi}$ (see Lemma $1(\mathrm{a})), \tilde{\phi}>|\phi|$ on some neighbourhood of $z_{0}$, hence by the symmetry and strict monotonicity of $V, V(\tilde{\phi})>V(\phi)$ on this neighbourhood, and thus $U(\tilde{q})>U(q)$. Consequently, there exists $\lambda \in(0,1)$ such that $U(\lambda \tilde{q})=U(q)$, so that $\lambda \tilde{q} \in \mathscr{C}_{K}$. But since (by the definition of $\left.\tilde{q}\right) T(\tilde{q})=T(q)$, we compute

$$
T(\lambda \tilde{q})=\lambda^{2} T(\tilde{q})<T(\tilde{q})=T(q),
$$

contradicting the fact that $q$ is a minimiser. This establishes (25). Now suppose $q$ was not monotone, i.e. suppose there exist $a, b \in \mathbb{R}$ such that $q^{\prime}(a)<0, q^{\prime}(b)>0$. (Note that by Lemma $6, q^{\prime}$ is not just an $L^{2}$ function but a continuous function, so it makes sense to speak of its pointwise values.) By (25),

$$
\int_{z}^{z+1}\left|q^{\prime}\right|=\left|\int_{z}^{z+1} q^{\prime}\right| \text { for all } z \in \mathbb{R}
$$

thus on each interval $[z, z+1]$ of length one, either $q^{\prime} \geq 0$ or $q^{\prime} \leq 0$. Therefore, there exist $z_{1}, z_{2} \in(a, b)(\in(b, a)$ in case $b<a)$ with $\left|z_{2}-z_{1}\right| \geq 1,\left.\overline{q^{\prime}}\right|_{\left[z_{1}, z_{2}\right]} \equiv 0$. Choose $z_{1}, z_{2}$ so that in addition

$$
\operatorname{dist}\left(z_{i},\left\{z \in \mathbb{R}: q^{\prime}(z) \neq 0\right\}\right)=0 \quad \text { for } \quad i=1,2 \text {. }
$$

Now consider the function

$$
\tilde{\tilde{q}}(z):= \begin{cases}\tilde{q}(z), & z \leq z_{1} \\ \tilde{q}\left(z+\left(z_{2}-z_{1}\right)\right), & z>z_{1}\end{cases}
$$


Since $\left|z_{2}-z_{1}\right| \geq 1$, we compute

$$
\begin{aligned}
U(\tilde{q})-U(\tilde{q})= & \int_{z_{1}-1}^{z_{1}} V\left(\tilde{q}\left(z+1+\left(z_{2}-z_{1}\right)\right)-\tilde{q}(z)\right) \\
& -\int_{z_{1}-1}^{z_{1}} V\left(\tilde{q}\left(z_{1}\right)-\tilde{q}(z)\right)-\int_{z_{2}-1}^{z_{2}} V\left(\tilde{q}(z+1)-\tilde{q}\left(z_{1}\right)\right) \\
= & \int_{z_{1}-1}^{z_{1}}\left(V\left(r_{1}(z)+r_{2}(z)\right)-V\left(r_{1}(z)\right)-V\left(r_{2}(z)\right)\right)
\end{aligned}
$$

with $r_{1}(z)=\tilde{q}\left(z_{1}\right)-\tilde{q}(z)(\geq 0), r_{2}(z)=\tilde{q}\left(z+1+\left(z_{2}-z_{1}\right)\right)-\tilde{q}\left(z_{1}\right)(\geq 0)$. Now by the superquadraticity of $V, V\left(r_{1}+r_{2}\right) \geq V\left(r_{1}\right)+V\left(r_{2}\right)$ for all $r_{1}, r_{2} \geq 0$, with equality if and only if one of the $r_{i}$ is zero. By (26), there exists $z \in\left[z_{1}-1, z_{1}\right]$ such that both $r_{1}(z)>0$ and $r_{2}(z)>0$ hence $U(\tilde{q})>U(\tilde{q})$. We now arrive at a contradiction, by arguing as in the proof of (25): $U(\lambda \tilde{\tilde{q}})=U(\tilde{q})$ for some $\lambda \in(0,1)$, and

$$
T(\lambda \tilde{\tilde{q}})=\lambda^{2} T(\tilde{\tilde{q}})<T(\tilde{\tilde{q}})=T(\tilde{q})=T(q),
$$

contradicting the fact that $q$ is a minimiser. The proof of the lemma is complete. As a corollary, we can finally prove our existence theorem:

Proof of Theorem 1. Applying Propositions 2 and 3 and Remark 1 to the Hamiltonian with symmetrised interaction potential $\tilde{V}$ (as defined in (P4)) gives existence of minimisers $q_{K}$ for the claimed ranges of $K$. If $q_{K}$ is a minimiser, then trivially so is $-q_{K}$, and by Lemma 7 , either $\tilde{V} \equiv V$ on range $\left(\phi_{K}\right)$ or $\tilde{V} \equiv V$ on range $\left(-\phi_{K}\right)$. Hence by Lemma 6 , either $q_{K}$ or $-q_{K}$ is a solution of (3), and satisfies (P1), (P4). (P2) follows from Lemma 1(a). Finally, (P3) is an easy consequence of (24) and the energy inequality (E), as follows. Since $\frac{V(r)}{r^{2}}$ increases with $|r|$, by differentiating we obtain

$$
\frac{1}{2} V^{\prime}(r) r \geq V(r) \quad \forall r \in \Lambda .
$$

The second component of formula (24) with $\lambda=c_{K}^{2}$, the above inequality, and (E) (which must hold by (19) and (6)) give

$$
c_{K}^{2}=\frac{\frac{1}{2} \int_{\mathbb{R}} V^{\prime}\left(\phi_{K}\right) \phi_{K}}{T\left(q_{K}\right)} \geq \frac{U\left(q_{K}\right)}{T\left(q_{K}\right)}>V^{\prime \prime}(0),
$$

completing the proof of the theorem.

\section{The Lennard-Jones Potential}

In this section we deal with singular potentials like the Lennard-Jones $(6,12)$ potential

$$
V(\phi)=\left\{\begin{array}{ll}
a\left((d+\phi)^{-12}-2 d^{-6}(d+\phi)^{-6}+d^{-12}\right), & \phi>-d \\
+\infty, & \phi \leq-d
\end{array} \quad(a, d>0),\right.
$$


describing the interaction between particles in the microscopic theory of classical fluids. Due to their singularity, these potentials do not fit directly into the framework of Theorem 1, but require a little extra work. The result of this section is

Corollary 1. Let $V(\phi)=\left\{\begin{array}{ll}V_{0}(\phi), & \phi>-d \\ +\infty, & \phi \leq-d\end{array}\right.$ for some $d>0$, and assume that $V_{0} \in C^{2}(-d, \infty), V_{0} \geq 0, V_{0}(0)=0, V_{0}$ superquadratic on $(-d, 0), V_{0}^{\prime \prime}(0) \neq 0$, and that hypothesis $(\mathrm{H} 2 \mathrm{~b})$ of Theorem 1 holds. Then for all $K \in\left(0, K_{0}\right)$ (and some $\left.K_{0} \geq \frac{1}{2} V^{\prime \prime}(0) d^{2}\right)$, the Hamiltonian system (1) admits a solitary wave $q_{K}$ with finite kinetic energy and average potential energy $K$ (i.e., there exists a solution $q_{K} \in C^{2}(\mathbb{R})$ of Eq. (2) with $\left.T\left(q_{K}\right)<\infty, U\left(q_{K}\right)=K\right)$, with the properties:

$$
-d<\phi_{K}(z) \leq 0 \text { for all } z \in \mathbb{R}
$$

(i.e. $q_{K}$ is a compression wave, and respects the physical constraint that interpenetration of matter is forbidden and the lattice sites stay ordered), and properties (P2), (P3), (P4) of Theorem 1.

In particular, the corollary applies to the Lennard-Jones potential (27), which was shown in Sect. 2 to be superquadratic on $(-d, 0)$ and which satisfies $(\mathrm{H} 2 \mathrm{~b})$ since $V^{\prime \prime \prime}(0)<0$.

Proof. The proof relies on a simple cutoff-argument which makes Theorem 1 applicable. Fix $\varepsilon \in\left(0, \frac{d}{2}\right)$, and choose a cutoff-function $\eta^{\varepsilon} \in C^{\infty}(\mathbb{R})$ with $\eta \equiv 0$ on $(-\infty,-d+\varepsilon), \eta \equiv 1$ on $(-d+2 \varepsilon, \infty), \eta^{\prime} \geq 0$. Let $p$ be the exponent from (H2b), and choose $C^{\varepsilon}$ so large that

$$
W^{\varepsilon}(r):=\frac{V^{\prime \prime}(0)}{2} r^{2}+C|r|^{p}>V(r) \text { for } r \in(-d+\varepsilon, 0),
$$

and define a smooth potential on the whole of $\mathbb{R}$ by

$$
V^{\varepsilon}(r):= \begin{cases}W^{\varepsilon}(r), & r \leq-d \\ \eta(r) V(r)+(1-\eta(r)) \cdot W^{\varepsilon}(r), & r>-d\end{cases}
$$

Using the superquadraticity of $V$ and $W$ and the fact that $\eta^{\prime} \cdot(V-W) \leq 0$ on $(-\infty, 0)$, one immediately checks $\left(\frac{V^{\varepsilon}(r)}{r^{2}}\right)^{\prime}<0(r<0)$, thus $V^{\varepsilon}$ is superquadratic on $(-\infty, 0)$. Clearly also $V^{\varepsilon}$ satisfies hypothesis $(\mathrm{H} 2 \mathrm{~b})$ of Theorem 1 , hence Theorem 1 applies and for all $K>0$ we obtain existence of solutions $q_{K}^{\varepsilon}$ to the equation

$$
c^{2} q^{\prime \prime}(z)=\left(V^{\varepsilon}\right)^{\prime}(q(z+1)-q(z))-\left(V^{\varepsilon}\right)^{\prime}(q(z)-q(z-1)),
$$

which have average potential energy $U^{\varepsilon}\left(q_{K}^{\varepsilon}\right)=K$. Since $V^{\varepsilon} \equiv V$ on $(-d+2 \varepsilon, \infty)$, it only remains to prove that for $K<\frac{1}{2} V^{\prime \prime}(0) d^{2}$ and appropriately chosen $\varepsilon$, $\phi_{K}^{\varepsilon}(z)>-d+2 \varepsilon$ for all $z \in \mathbb{R}$. Now by Lemma 1(a) and the energy inequality (E), we can bound the supremum of $\left|\phi_{K}^{\varepsilon}(z)\right|$ :

$$
\sup _{z \in \mathbb{R}}\left|\phi_{K}^{\varepsilon}(z)\right| \leq\left\|q_{K}^{\varepsilon}\right\|=\left(2 T_{K}^{\varepsilon}\right)^{1 / 2}<\left(\frac{2 K}{V^{\prime \prime}(0)}\right)^{1 / 2}
$$

hence given $K<\frac{1}{2} V^{\prime \prime}(0) d^{2}$ pick $\varepsilon \leq \frac{1}{2}\left(d-\left(\frac{2 K}{V^{\prime \prime}(0)}\right)^{1 / 2}\right)$, then $\phi_{K}^{\varepsilon}(z)>-d+2 \varepsilon$ for all $z$, as required. The corollary is proved. 


\section{Nonexistence for Quadratic Potentials}

For the convenience of the reader, we include a brief rigorous proof of the well-known fact (which complements our existence theory above) that in the case of a quadratic potential $V$, no solitary waves exist.

Lemma 8. Let $V(r)=\frac{a}{2} r^{2}(a \in \mathbb{R})$. Then Eq. (3) does not admit any nonconstant solutions $q \in C^{2}(\mathbb{R})$ with finite kinetic energy $T(q)<\infty$.

Proof. Via the substitution $\phi(\cdot)=q(\cdot+1)-q(\cdot)$, Eq. (3) transforms into

$$
c^{2} \phi^{\prime \prime}=V^{\prime}\left(\phi_{+}\right)-2 V^{\prime}(\phi)+V^{\prime}\left(\phi_{-}\right),
$$

where $\phi_{ \pm}=\phi(\cdot \pm 1)$. Now by (6), solutions $q$ of (3) with finite kinetic energy correspond to square-integrable solutions $\phi$ of (28), and can thus be found using the Fourier transform (which is well-defined as a mapping $\left(L^{2}(\mathbb{R}) \rightarrow L^{2}(\mathbb{R})\right)$ : if $\phi$ solves (28) and $\hat{\phi}$ denotes its Fourier transform,

$$
\hat{\phi}(k)=(2 \pi)^{-1 / 2} \int_{\mathbb{R}} e^{-\imath k z} \phi(z) d z,
$$

then $-k^{2} c^{2} \hat{\phi}(k)=2(\cos k-1) a \hat{\phi}(k)$, hence $\hat{\phi}(k)=0$ almost everywhere, and thus $\phi \equiv 0$, implying $q \equiv$ const. This proves the lemma. Note that if instead of finite energy waves $\phi \in L^{2}(\mathbb{R})$, we allow waves whose Fourier transforms are distributions, the above equation admits solutions $\hat{\phi}$, consisting of finite sums of Dirac delta-functions; then $\phi(z)$ is a superposition of linear waves, i.e. it will be a quasi-periodic delocalised wave, and consequently have infinite energy.

\section{Finite Lattice}

On a finite lattice with $N$ particles, having the Hamiltonian

$$
H=\sum_{n=1}^{N} \frac{1}{2} p_{n}^{2}+V\left(q_{(n+1) \bmod N}-q_{n}\right),
$$

our variational formulation of the question of existence of travelling waves can of course also be used. In this case we minimise $T(q)=\int_{0}^{N} q^{\prime}(z)^{2} d z$ on the Hilbert space

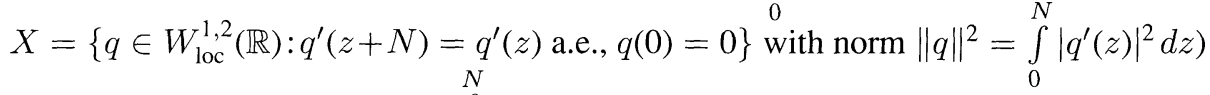
subject to the condition $U(q)=\int_{0}^{N} V(q(z+1)-q(z)) d z=K$. Due to the boundedness of the domain, here the constraint is weakly continuous on $X$ (for arbitrary potentials $V \in C^{0}(\mathbb{R})$ ), hence unlike in the case of an infinite domain, existence of minimisers (for any $K$ such that $\mathscr{A}_{K}:=\{q \in X: U(q)=K\}$ is nonempty) is straightforward. So we only have to assume $V \in C^{1}(\mathbb{R}), V(0)=0, V(r)$ increasing (decreasing) with $r>0(r<0)$ and $V(r) \rightarrow \infty$ as $|r| \rightarrow \infty$ (to guarantee $\mathscr{t}_{K}$ nonempty for all $K>0$ ), and obtain existence of a travelling wave with potential energy $K$ for all $K>0$, regardless of whether the interaction potential is super- or subquadratic. (A similar argument has in fact already been used in [21] to establish existence of periodic travelling waves for a class of Lennard-Jones type potentials.) Note, however, 
that these simple arguments give no indication of how localised these travelling waves are for large $N$, and do not detect the "phase transition" seen on the infinite lattice when passing from superquadratic to subquadratic nonlinearities.

Acknowledgements. We thank J. M. Ball and J. C. Eilbeck for continuous advice and encouragement.

\section{References}

1. Eilbeck, J.C., Flesch, R.: Calculation of families of solitary waves on discrete lattices. Phys. Lett. A 149, 200-202 (1990)

2. Fermi, E., Pasta, J., Ulam, S.: Studies of nonlinear problems. Los Almos Scientific Laboratory report LA-1940, 1955. Reprinted in: Lect. in Appl. Math. 15, 143-156 (1974)

3. Lions, P.L.: The concentration-compactness principle in the calculus of variations. The locally compact case, Part 1. Ann. Inst. Henri Poincaré 1, 109-145 (1984)

4. Flytzanis, N., Pnevmatikos, S., Peyrard, M.: Discrete lattice solitons: properties and stability. J. Phys. A 22, 783-801 (1989)

5. Peyrard, M., Pnevmatikos, S., Flytzanis, N.: Discreteness effects on non-topological kink soliton dynamics in nonlinear lattices. Physica D 19, 268-281 (1986)

6. Rosenau, P.: Dynamics of nonlinear mass spring chains near the continuum limit. Phys. Lett. A 118, 222-227 (1986)

7. Wattis, J.A.D.: Approximations to solitary waves on lattices. II. Quasi-continuum approximations for fast and slow waves. J. Phys. A 26, 1193-1209 (1993)

8. Hochstrasser, D., Mertens, F.G., Buttner, H.: An iterative method for the calculation of narrow solitary excitations on atomic chains. Physica D 35, 259-266 (1989)

9. Toda, M.: Theory of nonlinear lattices. Vol. 20 of Springer Series in Solid State Sciences. Berlin, Heidelberg, New York: Springer 1978

10. Eilbeck, J.C.: Numerical studies of solitons on lattices. In: Remoissenet, M., Peyrard, M. (eds.), Nonlinear Coherent Structures in Physics and biology. In: Lect. Notes in Phys. Vol. 393, Berlin, Heidelberg, New York: Springer 1991, pp. 143-150

11. Zabusky, N.J., Kruskal, M.D.: Interaction of "solitons" in a collisionless plasma and the recurrence of initial states. Phys. Rev. Lett. 15, 240-243 (1965)

12. Gardner, C.S., Greene, J.M., Kruskal, M.D., Miura, R.M.: Method for solving the Korteweg de Vries equation. Phys. Rev. Lett. 19, 1095-1097 (1967)

13. Sholl, D.S., Henry, B.I.: Perturbative calculation of superperiod recurrence times in nonlinear chains. Phys. Lett. A 159, 21-27 (1991). Note p. 26, top of column 2

14. Duncan, D.B., Wattis, J.A.D.: Approximations to solitary waves on lattices, use of identities and the lagrangian formulation. Chaos, Solitons and Fractals 2, 505-518 (1992)

15. Russell, S.: Report on waves. Report of the $14^{\text {th }}$ Meeting of the British Association for the Advancement of Science, 1844 pp. 311-390

16. Flytzanis, N., Malomed, B.A., Wattis, J.A.D.: Analaysis of stability of solitons in onedimensional lattices. Phys. Lett. A 180, 107-112 (1993)

17. Ball, J.M.: Loss of the constraint in convex variational problems. In: Analyse Mathématique et Applications. Paris: Gauthier-Villars 1988, pp. 39-53

18. Ball, J.M., Carr, J., Penrose, O.: The Becker-Döring cluster equations: Basic properties and asymptotic behaviour of solutions. Commun. Math. Phys. 104, 657-692 (1986)

19. Lieb, E.H., Simon, B.: The Thomas-Fermi theory of atoms, molecules, and solids. Adv. Math. 23, 22-116 (1977)

20. Cazenave, T., Lions, P.L.: Orbital stability of standing waves for some nonlinear Schrödinger equations. Commun. Math. Phys. 85, 549-561 (1982)

21. Valkering, T.P.: Periodic permanent waves in an anharmonic chain with nearest neighbour interactions. J. Phys. A 11, 1885-1897 (1978) 